Original paper

\title{
Artificial intelligence applications in medical imaging: A review of the medical physics research in Italy
}

\author{
Michele Avanzo ${ }^{\mathrm{a}, *}$, Massimiliano Porzio ${ }^{\mathrm{b}}$, Leda Lorenzon ${ }^{\mathrm{c}}$, Lisa Milan ${ }^{\mathrm{d}}$, Roberto Sghedoni ${ }^{\mathrm{e}}$, \\ Giorgio Russo ${ }^{\mathrm{f}}$, Raffaella Massafra ${ }^{\mathrm{g}}$, Annarita Fanizzi ${ }^{\mathrm{g}}$, Andrea Barucci ${ }^{\mathrm{h}}$, Veronica Ardu ${ }^{\mathrm{i}}$, \\ Marco Branchini ${ }^{j}$, Marco Giannelli ${ }^{k}$, Elena Gallio ${ }^{1}$, Savino Cilla ${ }^{\mathrm{m}}$, Sabina Tangaro ${ }^{\mathrm{n}, \mathrm{o}}$, \\ Angela Lombardi ${ }^{\mathrm{o}, \mathrm{p}}$, Giovanni Pirrone ${ }^{\mathrm{a}}$, Elena De Martin ${ }^{\mathrm{q}}$, Alessia Giuliano ${ }^{\mathrm{r}}$, Gina Belmonte ${ }^{\mathrm{r}}$, \\ Serenella Russo $^{\mathrm{s}}$, Osvaldo Rampado ${ }^{1}$, Giovanni Mettivier ${ }^{\mathrm{t}}$ \\ ${ }^{a}$ Medical Physics Department, Centro di Riferimento Oncologico di Aviano (CRO) IRCCS, 33081 Aviano PN, Italy \\ ${ }^{\mathrm{b}}$ Medical Physics Department, ASL CN1, Via Carlo Boggio 12, 12100 Cuneo CN, Italy \\ ${ }^{\mathrm{c}}$ Medical Physics Department, Bolzano Hospital, 39100 Bolzano, Italy \\ ${ }^{\mathrm{d}}$ Clinic of Nuclear Medicine and Molecular Imaging, Imaging Institute of Southern Switzerland, Ente Ospedaliero Cantonale, Bellinzona, Switzerland \\ ${ }^{\mathrm{e}}$ Medical Physics Unit, Azienda USL-IRCCS di Reggio Emilia, Via Amendola, 2, 42122 Reggio Emilia, Italy \\ ${ }^{\mathrm{f}}$ Secondary Site Cefalù - Institute of Bioimaging and Molecular Physiology (IBFM) - National Research Council (CNR), Laboratori Nazionali del Sud - National Institute \\ for Nuclear Physics (LNS-INFN) \\ ${ }^{g}$ Struttura Semplice Dipartimentale di Fisica Sanitaria, IRCCS. Istituto Tumori “Giovanni Paolo II", Bari, Italy \\ ${ }^{\mathrm{h}}$ Institute of Applied Physics "Nello Carrara", Italian National Research Council, via Madonna del Piano 10, Sesto Fiorentino, FI 50019, Italy \\ ${ }^{\mathrm{i}}$ UOS Fisica Sanitaria, ASST Lodi, Lodi, Italy \\ ${ }^{\mathrm{j}}$ Medical Physics Department, ASST Valtellina e Alto Lario, Via Stelvio 25, 23100 Sondrio, Italy \\ ${ }^{\mathrm{k}}$ Unit of Medical Physics, Pisa University Hospital “Azienda Ospedaliero-Universitaria Pisana", via Roma 67, 56126 Pisa, Italy \\ ${ }^{1}$ Medical Physics Unit, A.O.U. Città della Salute e della Scienza di Torino, 10126 Torino, Italy \\ ${ }^{\mathrm{m}}$ Medical Physics Unit, Gemelli Molise Hospital, Catholic University of Sacred Heart, 86100 Campobasso, Italy \\ n Dipartimento di Scienze del Suolo, della Pianta e degli Alimenti - Università degli Studi di Bari Aldo Moro, Via G. Amendola, 165/a, 70126 Bari, Italy \\ ${ }^{\circ}$ Istituto Nazionale di Fisica Nucleare - Sezione di Bari, Via Orabona 4, 70125 Bari, Italy \\ ${ }^{\mathrm{p}}$ Dipartimento Interateneo di Fisica, Università degli Studi di Bari Aldo Moro, Via Orabona 4, 70125 Bari, Italy \\ ${ }^{\mathrm{q}}$ Health Department, Fondazione IRCCS Istituto Neurologico Carlo Besta, 20133 Milan, Italy \\ ${ }^{\mathrm{r}}$ Medical Physics Department, San Luca Hospital, 55100 Lucca, Italy \\ ${ }^{\mathrm{s}}$ Medical Physics Unit, AUSL Toscana Centro, Via dell'Antella 58, 50012 Bagno a Ripoli (FI), Italy \\ ${ }^{\mathrm{t}}$ Dipartimento di Fisica E.Pancini, Università degli Studi di Napoli Federico II and INFN Napoli, I-80126 Napoli, Italy
}

\section{A R T I C L E I N F O}

\section{Keywords:}

Machine learning

Deep learning

Artificial intelligence

Imaging

Radiomics

Radiotherapy

\begin{abstract}
A B S T R A C T
Purpose: To perform a systematic review on the research on the application of artificial intelligence (AI) to imaging published in Italy and identify its fields of application, methods and results.

Materials and Methods: A Pubmed search was conducted using terms Artificial Intelligence, Machine Learning, Deep learning, imaging, and Italy as affiliation, excluding reviews and papers outside time interval 2015-2020. In a second phase, participants of the working group AI4MP on Artificial Intelligence of the Italian Association of Physics in Medicine (AIFM) searched for papers on AI in imaging.

Results: The Pubmed search produced 794 results. 168 studies were selected, of which 122 were from Pubmed search and 46 from the working group. The most used imaging modality was MRI (44\%) followed by CT(12\%) ad radiography/mammography (11\%). The most common clinical indication were neurological diseases $(29 \%)$ and diagnosis of cancer (25\%). Classification was the most common task for AI (57\%) followed by segmentation (16\%). $65 \%$ of studies used machine learning and 35\% used deep learning. We observed a rapid increase of research in Italy on artificial intelligence in the last 5 years, peaking at 155\% from 2018 to 2019.
\end{abstract}

\footnotetext{
* Corresponding author.

E-mail addresses: mavanzo@cro.it (M. Avanzo), a.barucci@ifac.cnr.it (A. Barucci).
} 
Conclusions: We are witnessing an unprecedented interest in AI applied to imaging in Italy, in a diversity of fields and imaging techniques. Further initiatives are needed to build common frameworks and databases, collaborations among different types of institutions, and guidelines for research on AI.

\section{Introduction}

Accurate and early diagnosis and prognosis are essential in many fields of healthcare. Artificial Intelligence (AI) applied to medical images allows for automated disease detection, characterization of histology, stage, or subtype, and patient classification according to therapy outcome or prognosis. It also permits outlining particular regions in the images, quantifying organ volumes, and extracting features from the images which, combined with machine learning algorithms, leads to quantification of image properties or image classification.

In recent years an unprecedented amount of digital imaging data has become available in medicine thanks to digitalization, affordable data storage, and improved imaging techniques. This leads to an unprecedented interest in applications of AI to images which has boosted research efforts of medical physicists (MPs) in Italy.

These efforts however, are more efficient when there is communication, collaboration, sharing of knowledge and common intents in the MP community. For these purposes, the Italian Association of Medical Physics (AIFM) [1] which is composed of 1284 medical physicists, has established the AI for Medical Physics (AI4MP) [2] task-group.

The purpose of this review, performed by the imaging subgroup of AI4MP, is to describe the status of the research in Italy on AI applied to imaging, by systematically analyzing research published in this field in Italy in the last 5 years. This work, besides providing an overview of the fields of application, methods for AI used and the results achieved, will serve to define future goals for the community of MP and facilitate research on AI applied to imaging by MPs in Italy.

\section{Machine learning and deep learning in imaging}

Machine learning (ML) is a field of AI algorithms (Fig. 1) which can recognize patterns in medical images by analyzing voxel intensity values or quantitative imaging features, called also "radiomic features", by identifying their best combination and building a model for classification or regression [3-6]. By ML, image features can also be combined with variables from other sources, such as dose distribution from the radiotherapy treatment [7]) or clinical variables [8] to improve accuracy of classification. Supervised ML is frequently employed in imaging for classification [7] when the output variable is categorical, and for

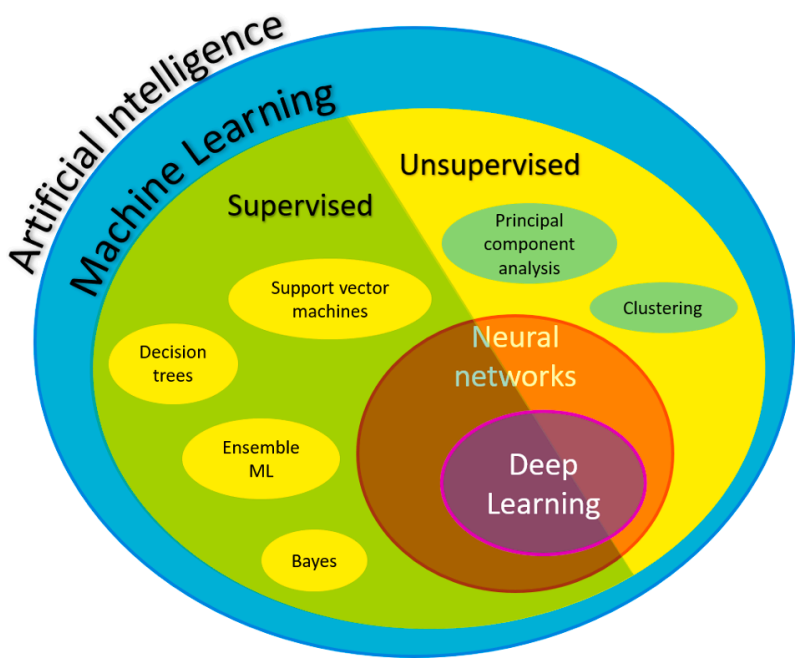

Fig. 1. Definitions of AI used in the review. regression [9] tasks when the output variable is continuous.

A large number of supervised ML algorithms is available, as shown in Table 1. Parametric algorithms make an assumption about the functional form of the function that map covariates to the outcome, then learn a finite number of coefficients for the function from the training data. These algorithms, by using a pre-selected function, are generally faster and easier to interpret. Generalized Linear Model with LASSO [9-12], Ridge or Elastic Net penalty and Logistic Regression (LR) belong to parametric ML. Non-parametric algorithms, by using a number of parameters which is not limited, are usually slower and require larger dataset. These include classification and Regression Trees (CART) $[13,14]$, K-Nearest Neighbours (KNN) $[15,16]$, and Support Vector Machines (SVM). SVM, based on finding a hyperplane that best divides the data into two classes in the feature space, is among most popular ML algorithm and is employed for both classification [7,10,17-20] and regression [16]. Stochastic search algorithms were developed in an effort to imitate the mechanics of natural selection and natural genetics [21].

Artificial neural networks (ANN) are often used in radiomics [22] for classification [23-25]. Random forest (RF), a popular concept in ML, are based on a large set of randomly generated decision trees which are trained individually. After training, the prediction is made for all the individual trees and the most frequently selected class is taken as a final result [26-30].

Unsupervised ML techniques can determine patterns in the data which can be used for cathegorization, without the need of userprovided labels. Examples are clustering methods such as k-means [31,32], hard C-means[33], hierarchical [34], and Fuzzy C-Means [35-38]. Less frequently, ML is employed for image segmentation using supervised [39-41] or unsupervised [21,35-38] e.g. by first computing features in the neighborhood of pixels which are then tagged using ML.

Deep learning (DL) [42-55]is a class of powerful MLs based on multiple deep layers of neural networks, characterized by hundreds of layers, each of which learns to detect different features of increasing complexity from an image. In contrast to ML, DL doesn't need to define $a$ priori a set of hand-crafted features, instead constructing its own internal features which are able to describe rich and comprehensive information, thus performing data representation and prediction jointly. CNNs can be used for regression [56-58], classification [59-61], segmentation [62-68] or image registration [58] tasks. Alternatively, DL networks can be used to extract machine learnt from their layers, which can then be passed to ML algorithms for classification $[9,69]$, or for image reconstruction [70].

Different DL architectures, such as Convolutional Neural Networks (CNN), Residual Networks (RN) [71], Autoencoders [72], Recurrent Neural Networks (RNN), allow for unlimited flexibility in extracting information (features and filters) outperforming humans in 2D/3D images analysis.

Instead of training a DL from scratch, architectures can be adapted from already existing and trained architecture such as Googlenet [56], Google Inception [73] or ENet [74]. The training an existing network by fine tuning its parameters and weights for a task which is unrelated to the present goal, called transfer learning, enables to develop efficiently accurate models $[56,75,76]$. RN are DL networks residual or skip connections, which jump some layers [69] making it possible to train deeper networks. Generative Adversarial Networks (GANs) transport the generative modeling approach in the context of DL. The idea behind this architecture is to learn to generate new data with the same statistics as the training set, using two neural networks contesting each other in a sort of a zero-sum game, where one agent's gain is another agent's loss. 


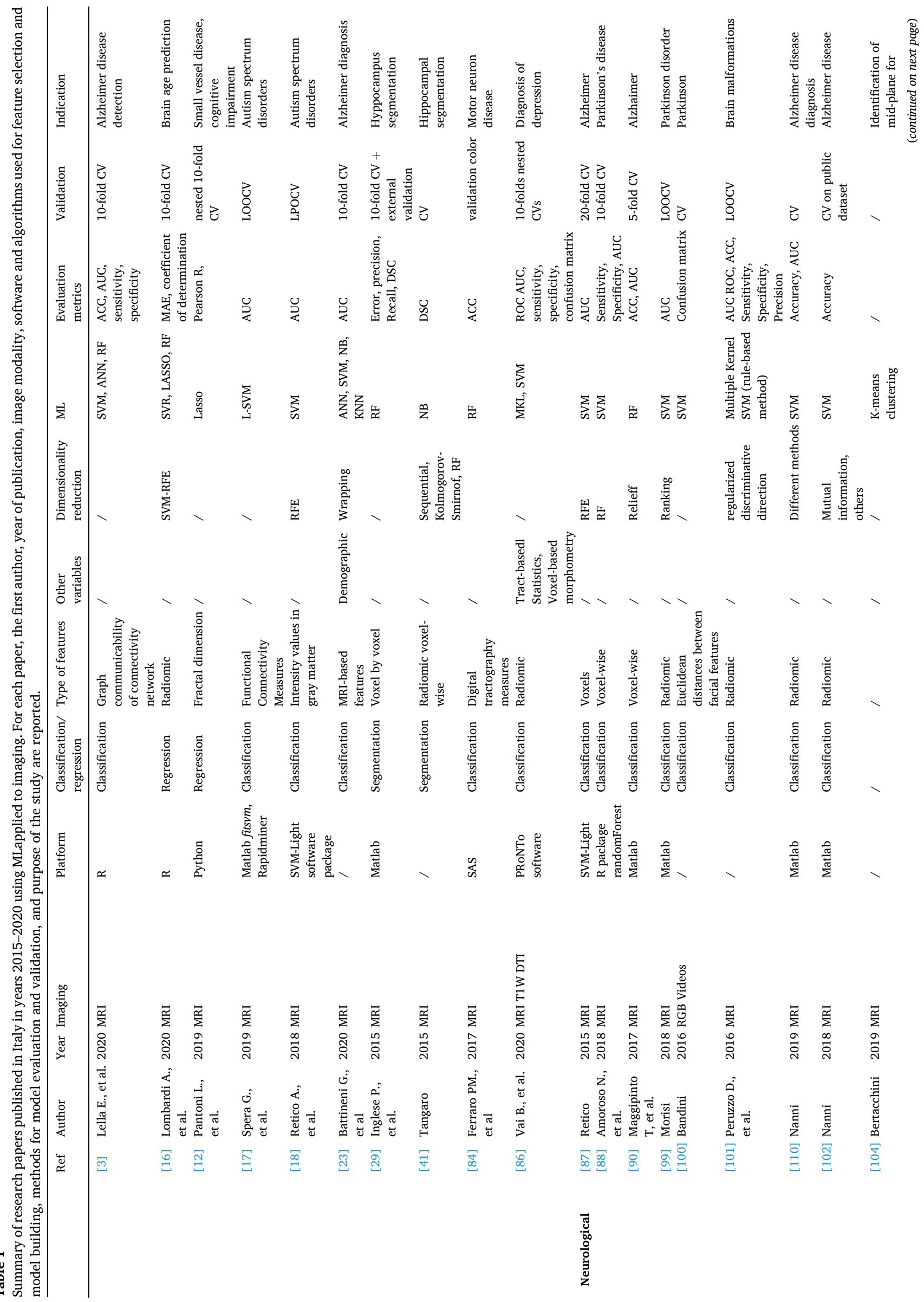




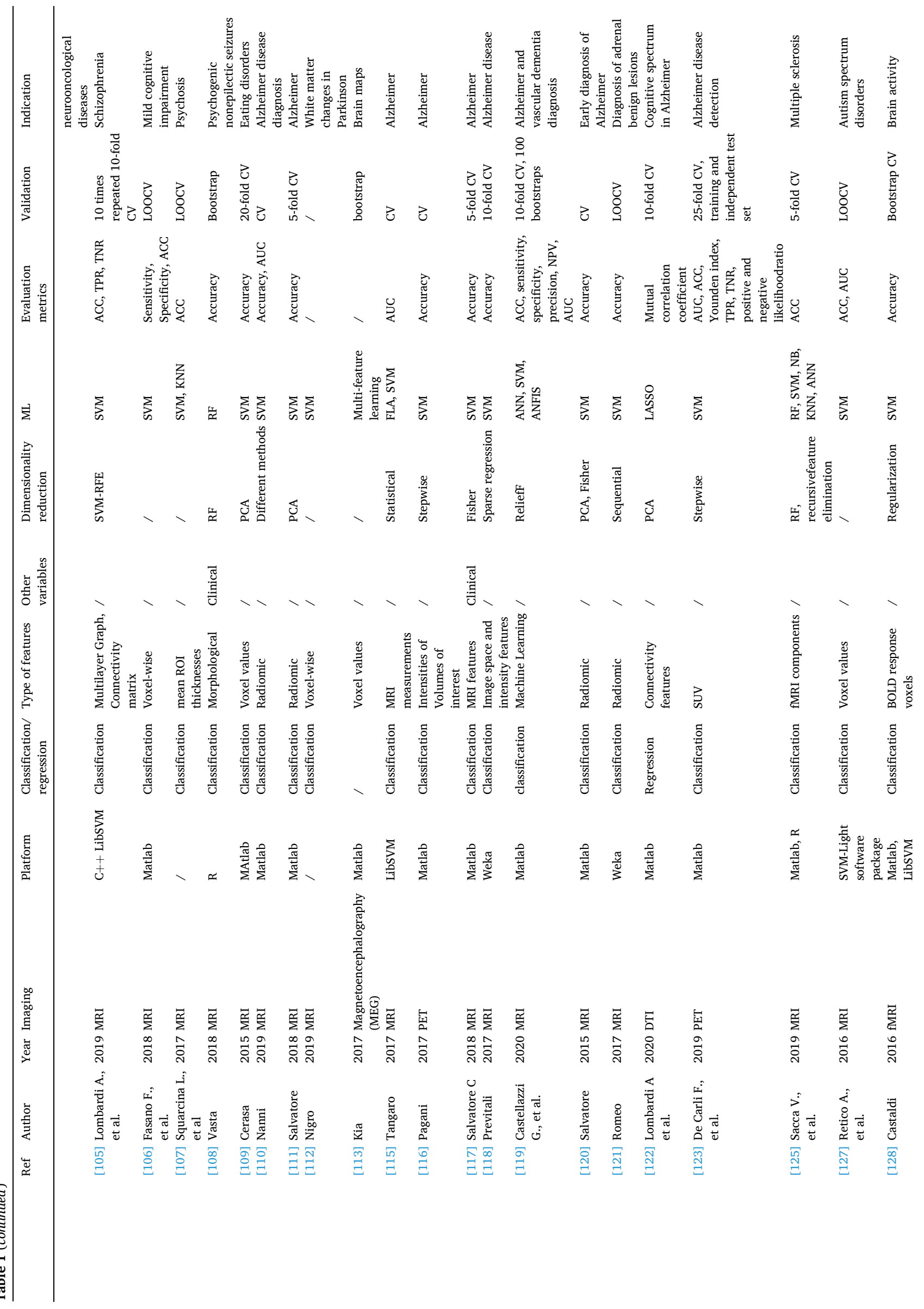




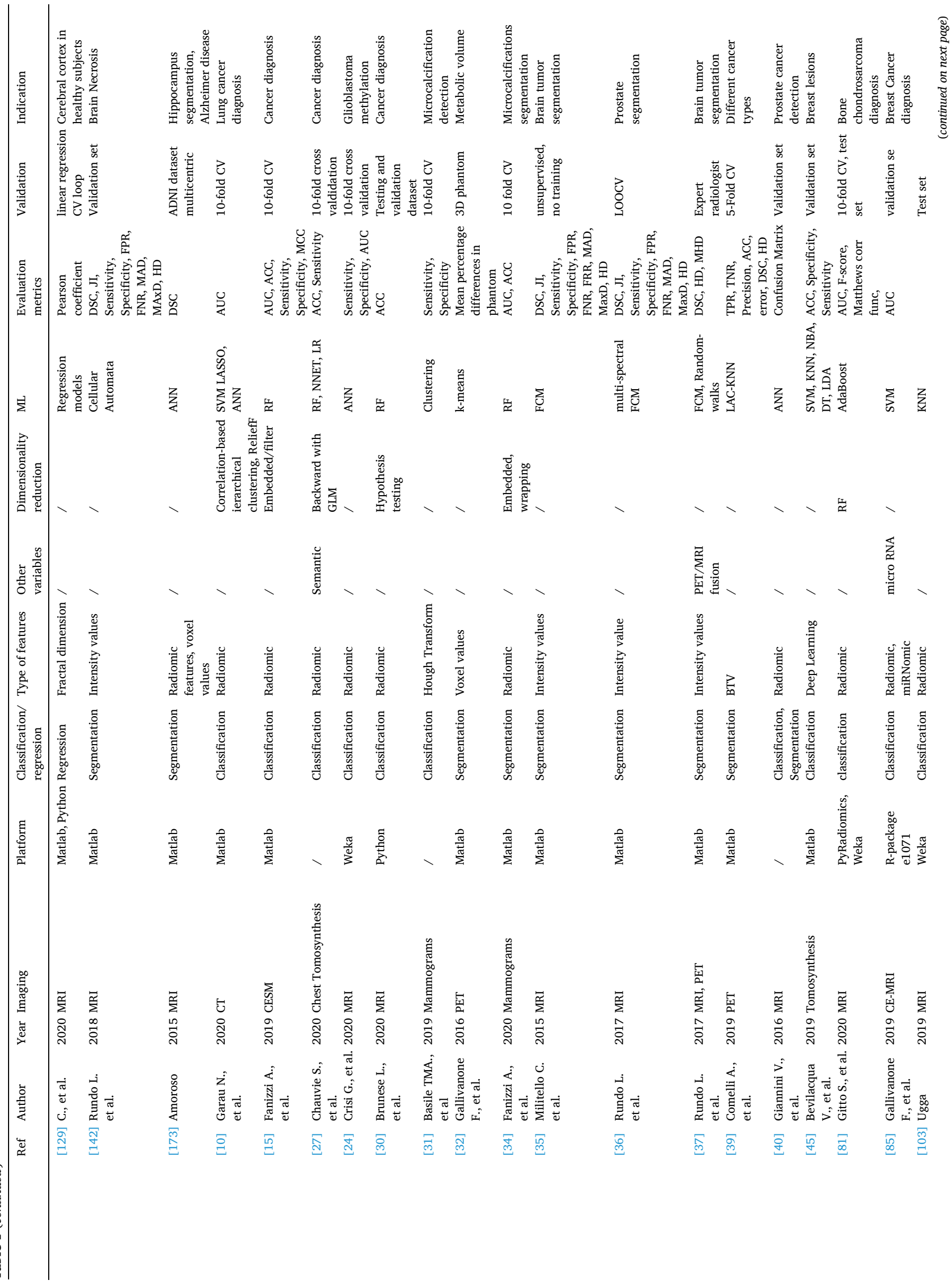




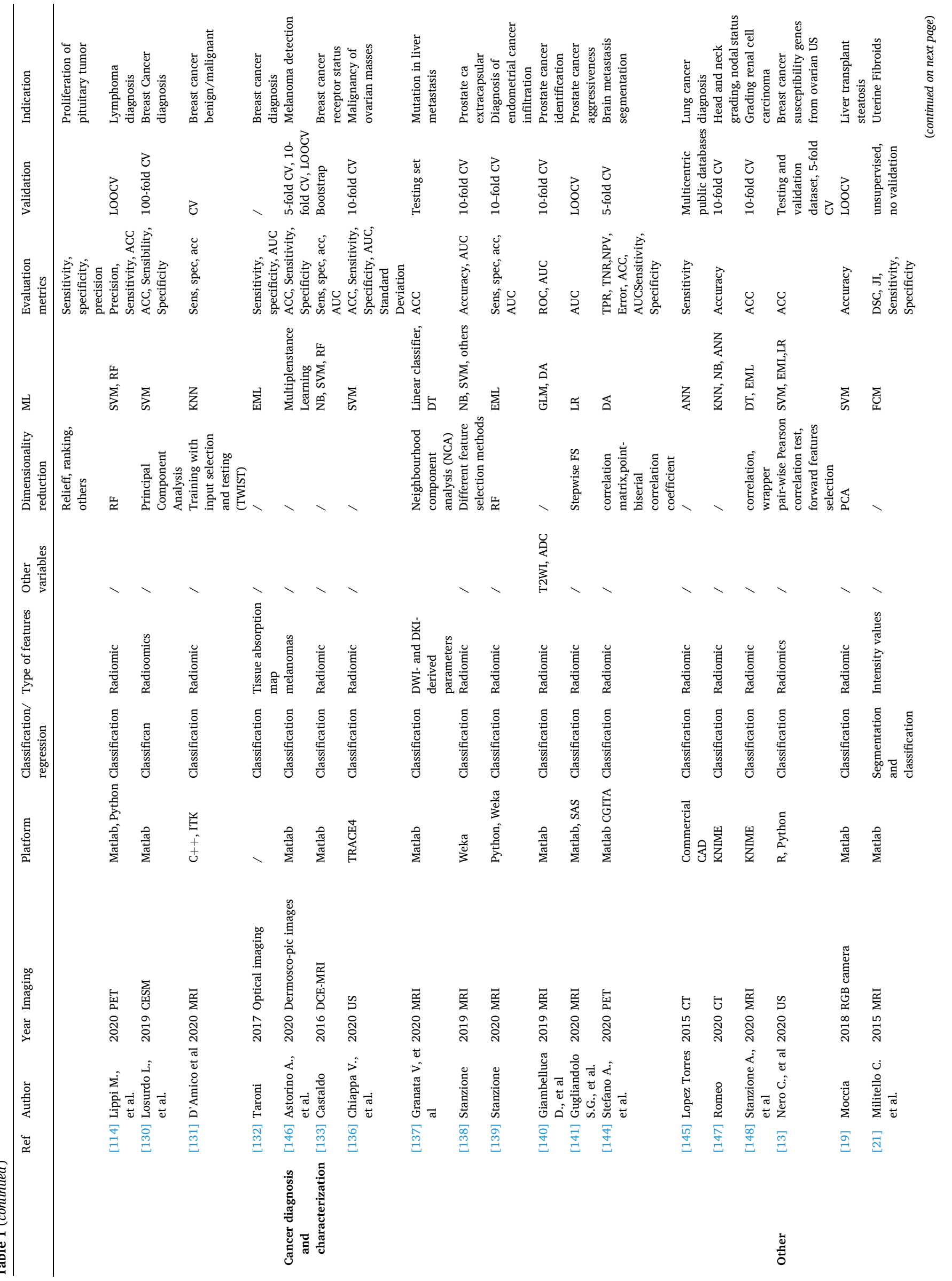




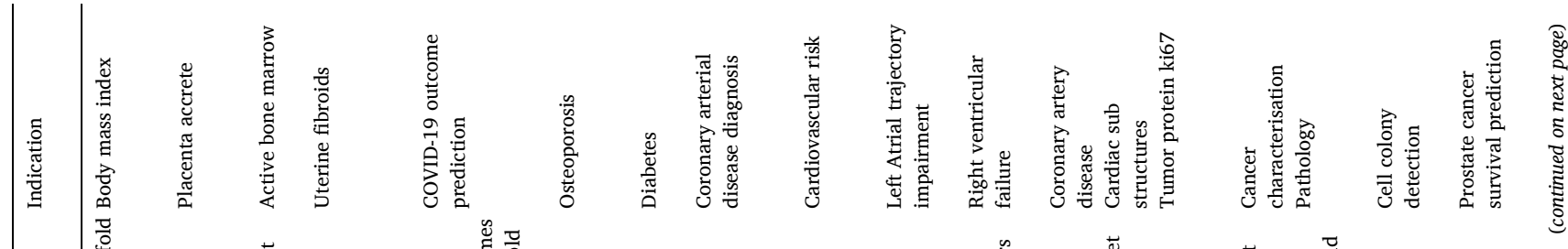

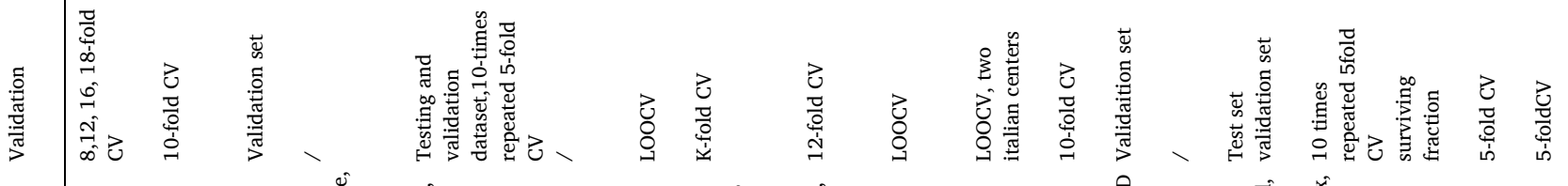

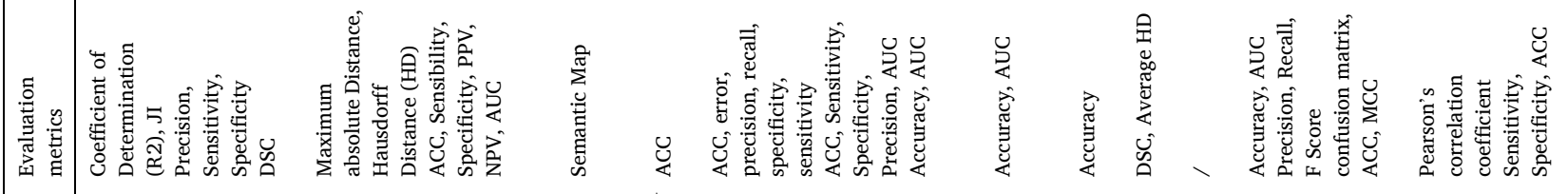

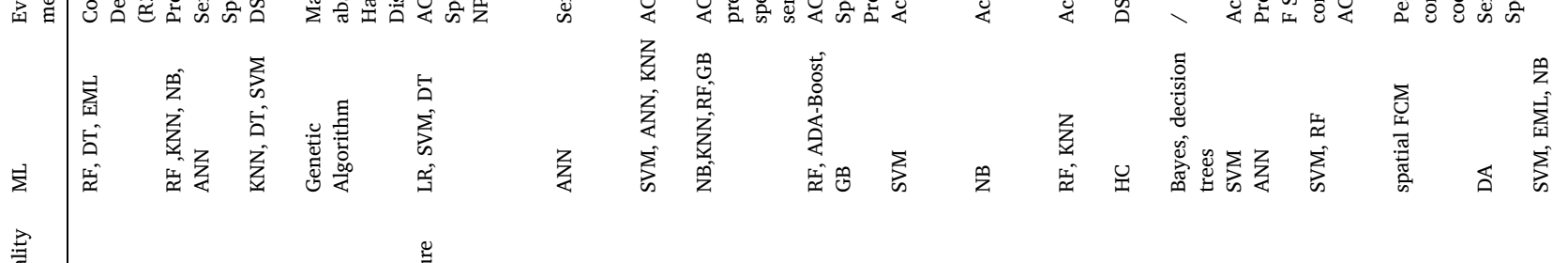
. . .<smiles>C=C(C=[IH])C1CC1</smiles>

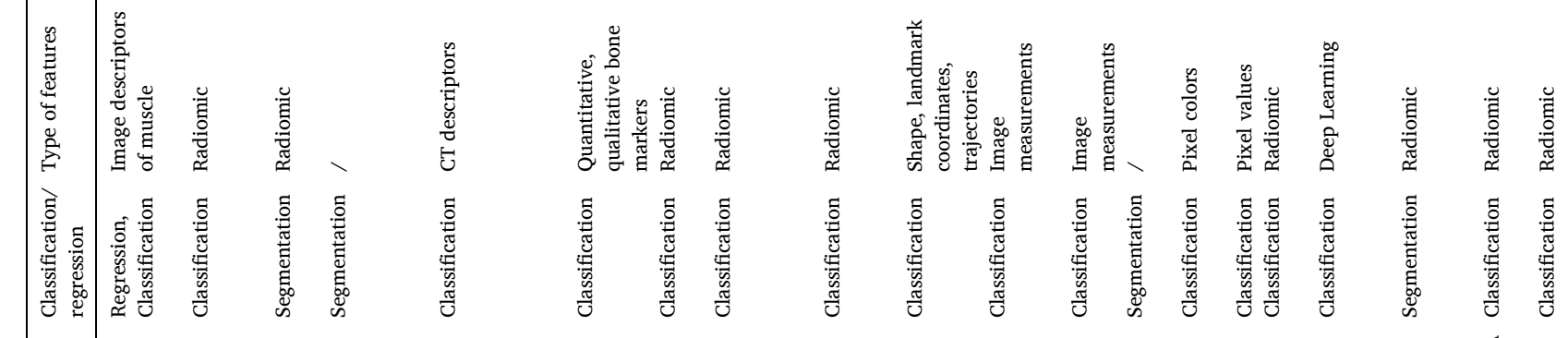

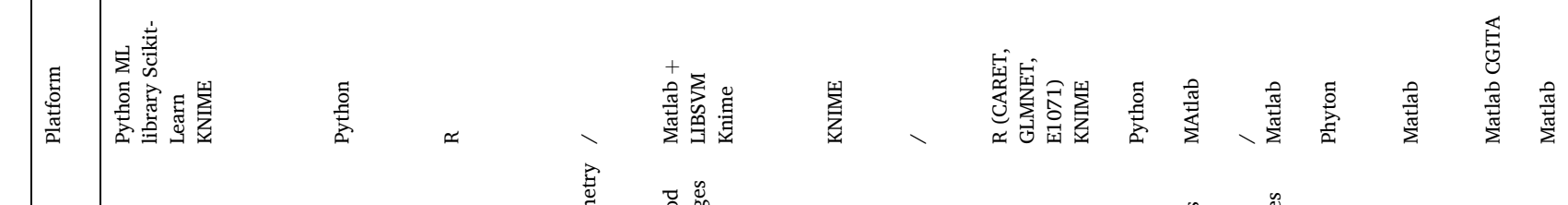

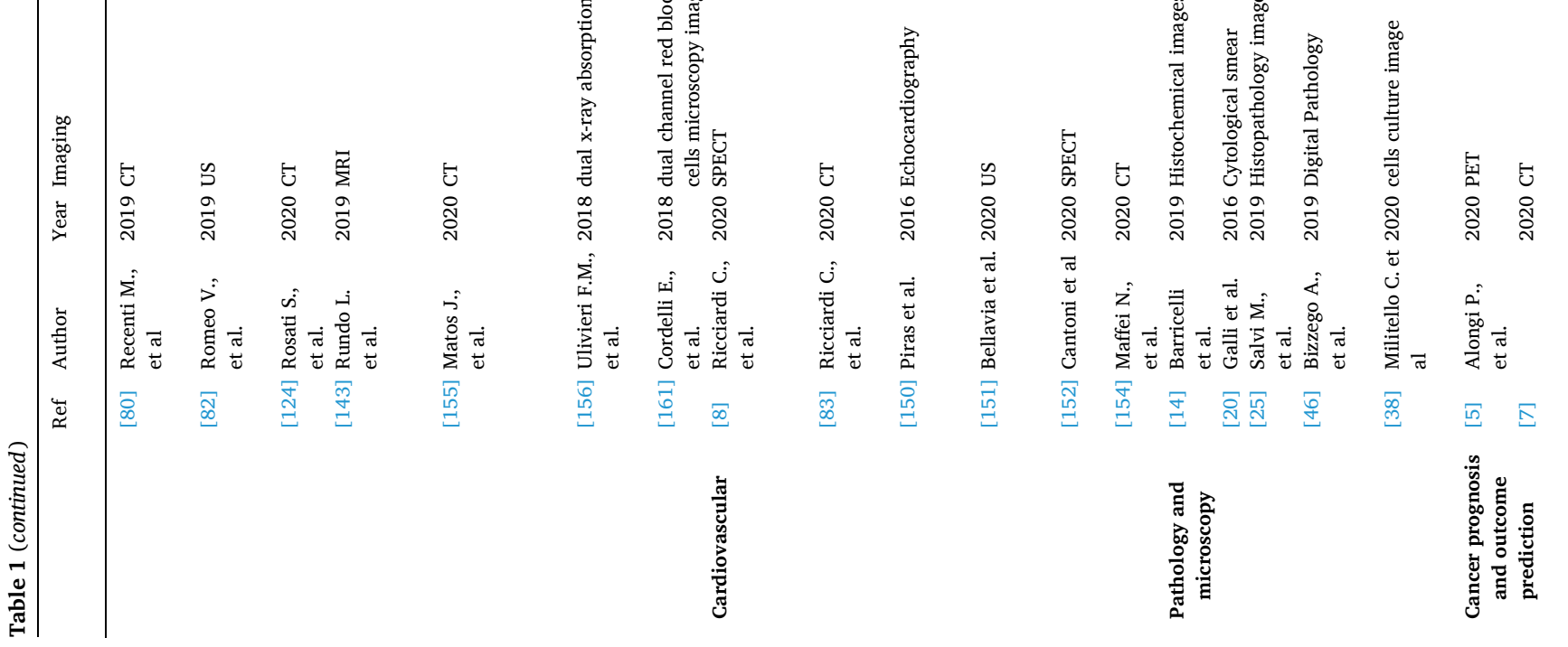




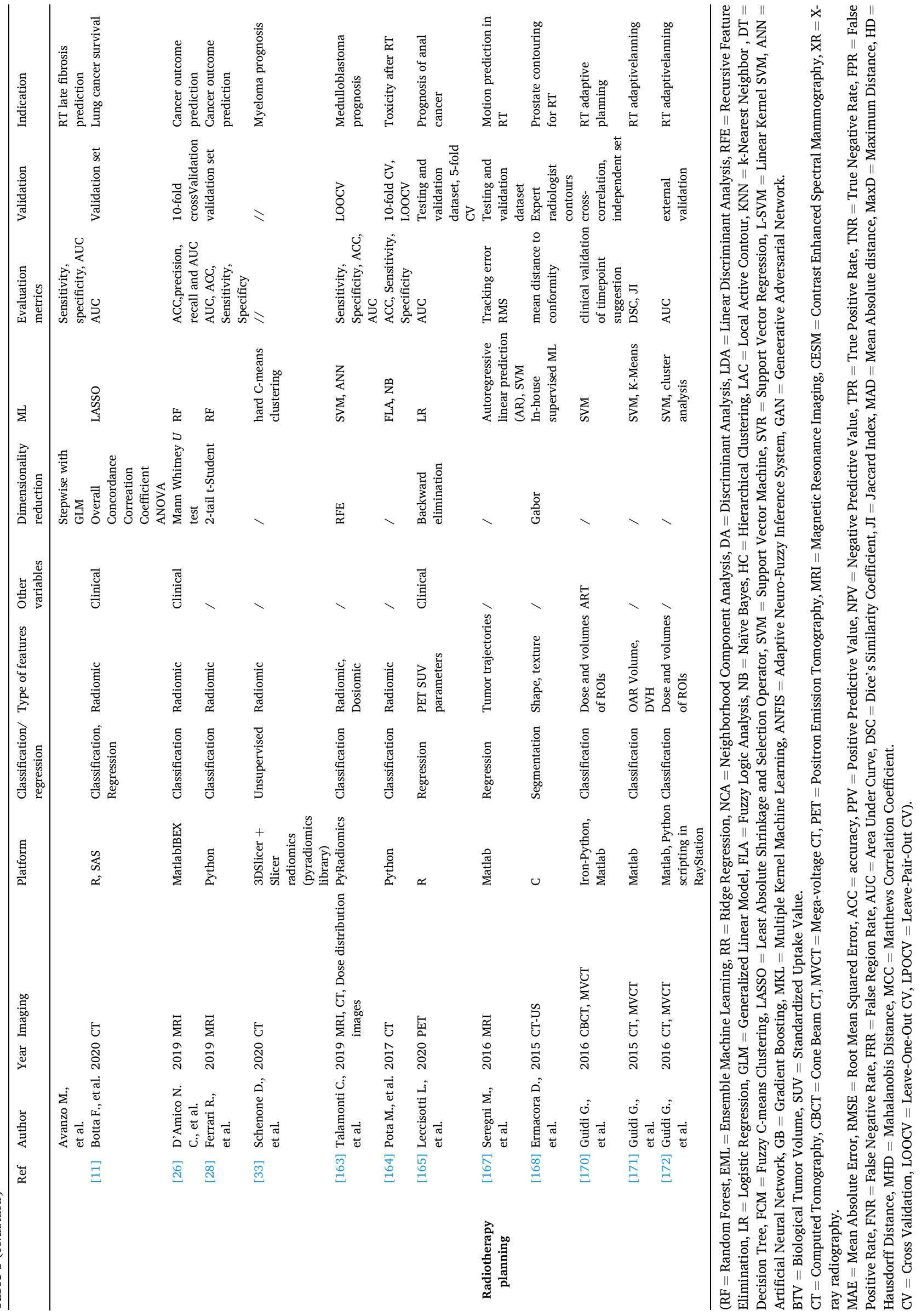


In the clinical context it is worth noting the use of GAN for Adversarial Learning, that is to build carefully targeted attacks to fool a model prediction [77-79].

\section{Systematic review}

A search for peer-reviewed manuscripts written in English was performed using the PubMed engine. The search was aimed at selecting studies performed in Italian institutions using machine learning applied to medical images. The search strategy was: "((((artificial intelligence) OR (machine learning)) OR (deep learning)) AND (imaging)) AND (italy [Affiliation])) NOT (review[Pubblication Type])", limited to years 2015-2020.

In a second phase, participants to the AI4FM task-group were asked to select the research papers, not covered in the first search, starting from those published from their institute on $\mathrm{AI}$ in imaging.

The participants independently reviewed the selected manuscripts. Studies were considered eligible when a machine learning method was applied to images or to features extracted from images, acquired for medical purpose, and the research was performed by investigators affiliated to insitutes in Italy.

For eligible studies, the AI method, type of AI task (classification, regression, segmentation, registration), algorithm, metric used for evaluation of AI performance, and type of validation, were collected. In order to study the type of institution were the research was conducted, the studies were categorized according to the affiliation of the first and last author, as hospital, university, public research institute (mainly National Institute for Nuclear Physics, INFN and National Research Council, CNR) or private company or foundation. The studies were considered as collaborations among different institutions of different types when the first and last authors came from different categories.

\section{Results of systematic review}

The search produced 793 papers. 120 studies were removed as not related to the medical field, 223 had not imaging or AI, 52 were editorials or reviews, 244 had not authors from Italy. 32 were removed as they had a significant overlap with other papers with higher impact factor by the same group. 122 were considered eligible, and 46 papers were manually retrieved by the participants in the group, for a total of 168 studies. These are shown in Table 1 (ML studies) and Table 2 (DL studies), were the clinical indication, algorithms and software platform used for each study are described.

We have observed a rapid increase of research in Italy on artificial intelligence in the last 5 years (Fig. 2). The increase in papers peaked at 155\% from 2018 to 2019.

Fig. 3a-c show the distribution of studies according to image modality, clinical indication, and institution were the research was undertaken.

Results show that most studies (36\%) were from universities followed by hospitals (16\%). The most used imaging modality was MRI (44\%), followed by CT(11\%) ad radiography/mammography (10\%). and the most common clinical indications were neurological diseases (29\%) and diagnosis of cancer (25\%).

Classification was the most common task for AI (57\%) followed by segmentation (16\%). Among ML algorithms, SVM was by far the most used ML (40.2\%). Most of the works retrieved use ML techniques (65\% of works).

\section{Used software tools}

Among the platforms used for ML (Table 1), the most used are Python ML specific libraries, like the Scikit-Learn library [80]. The Waikato Environment for Knowledge Analysis (Weka), is a free software developed at the University of Weikato, New Zealand, since 1993 [24,81]. Many ML algorithms from Weka are included in the Konstanz
Information Miner (Knime) analytics platform [8,82,83], where ML analyses are made intuitive by a graphical interface in which the tasks in the workflow (e.g. data reading, ML training and ML prediction) are visualised as nodes which can be configured and connected to each other. Commercial packages Matlab (Mathworks, Natick, MA), and SAS (SAS Institute, Cary, NC, USA) include ML and DL packages and graphical interfaces [84].

$\mathrm{R}$ also offers many R-package implementing most of the machine learning algorithms as glmnet for regularized generalized linear models, e1071 for SVM $[4,16,85]$, neuralnet for ANN, stats and kohonen for kmeans/hierarchical clustering and self-organizing maps. The caret package includes many algorithms implemented in different packages by specifying them as methods of a single training function. $\mathrm{H} 2 \mathrm{O}$ is a package for AI in R that offers parallelized implementations of ML [9]. PRoNTo (Pattern Recognition for Neuroimaging Toolbox) is a software toolbox providing analysis tools for neuroimaging including ML [86], while SVM-Light software is a package in C for SVM $[18,87]$.

Deep neural networks have been used to develop classification models on a variety of modalities including MRI [88,89], DTI [90], CT [91], PET [60], radiographs [92] as well as on videos [93] as shown in Table 2. Complex networks are graphs described by pairs of nodes and links that represent the elements of the system to be modelled and the iterations between the same, respectively, and allow to measure particularly informative topological features.

Mostly, DL (Table 2) have been used for End-to-End learning, i.e. networks learn how to do tasks automatically from raw data provided to only interested task, whereas 7 articles developed a DL model as features extractor for classification [45,46,59,61], regression [9,56-58], segmentation [62-67,74,77], or image registration [58]. Five works have used transfer learning to solve the task of interest $[42,56,75,76]$, e.g. for The Inception V3 and Alexnet pretrained on ImageNet large-scale database were used for grading of meningioma [44].

DL is mostly performed using the Python programming language and the Keras library $[69,57,70,75,94,95]$. Keras can be run as a stand-alone library or from TensorFlow (from tensorflow version greater than 2), an open-source software library for numeric computation [52]. The Google Colaboratory interface provides free parallel GPU computing for Python Jupyter notebooks. Theano [96], Caffe[97] and Pytorch [54,60,92,98] are other open source DL frameworks which can be run from Python.

\section{Clinical applications}

In the following sections we present the most representative studies on AI applied to imaging in Italy, grouped according to the clinical use of AI.

\section{Neurological applications}

The most common neuroimaging issues faced with AI tools are early diagnosis, biomarker identification and understanding the mechanism of development of neurodegenerative [99,100-102] and oncological diseases [103,104], mental disorders [101,105-109] and brain malformations [110]. Applications of AI in neuroimaging most commonly takes advantage of MRI, by applying ML to radiomic features ([111]) or directly to voxel intensity $[88,90,112,113]$.

Structural MRI, based on high resolution T1 weighted imaging (3DT1 MRI) has excellent contrast among soft tissues in brain, but diffusion tensor imaging (DTI) $[3,114]$ or resting-state functional MRI (rsfMRI), are increasingly used for characterizing the brain activity.

Accurate classification of Alzheimer disease (AD) and mild cognitive impairment (MCI) subjects has been achieved by combining novel topological descriptors and ML algorithms [115-121]. Volume descriptors or radiomic features [65] from MRI, alone or in combination with results from visuospatial tests were used in SVM classifiers to distinguish mild AD patients from healthy controls [76]. DL was applied to distinguish $\mathrm{AD}$ from $\mathrm{MCI}$ and predict which patients with $\mathrm{MCI}$ will develop $\mathrm{AD}$ 


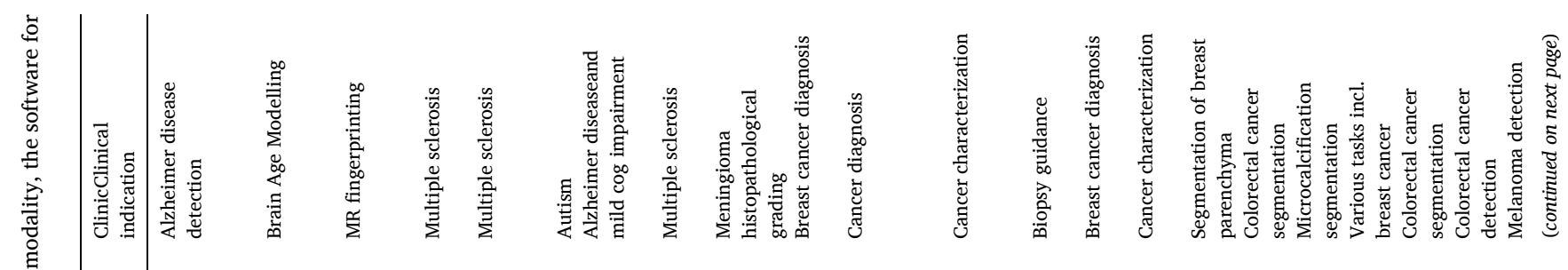

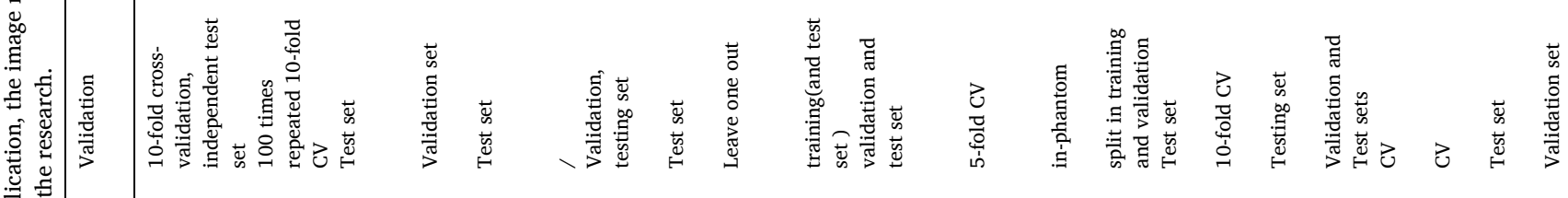

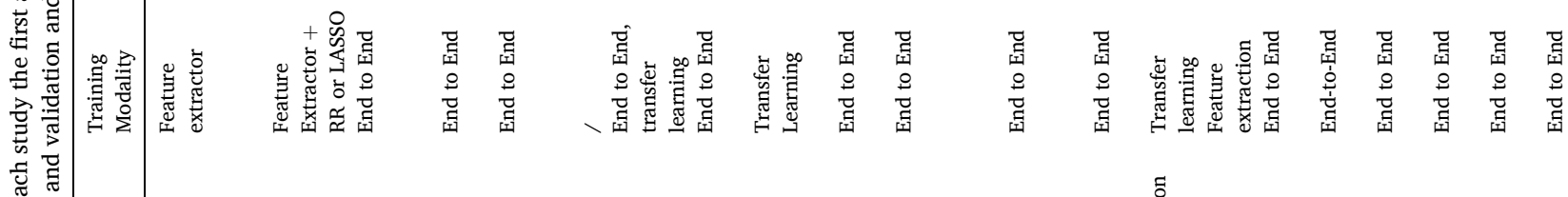

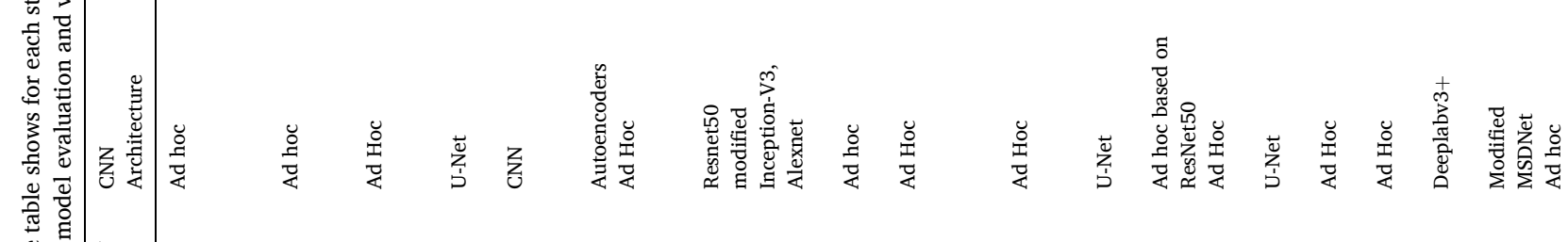

IIIIIIIIIIIIIIII IIIn

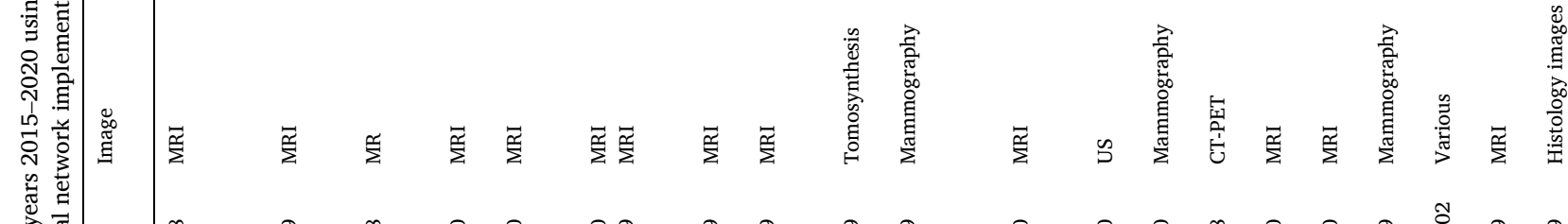

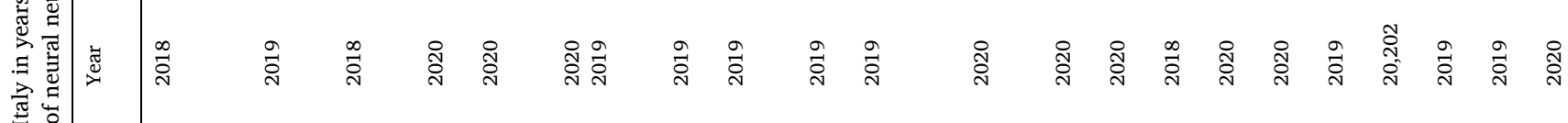

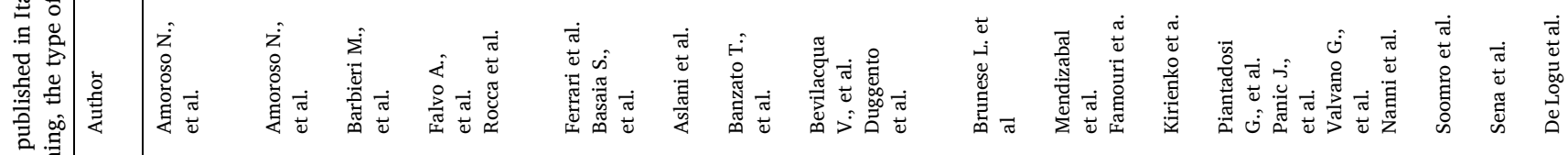

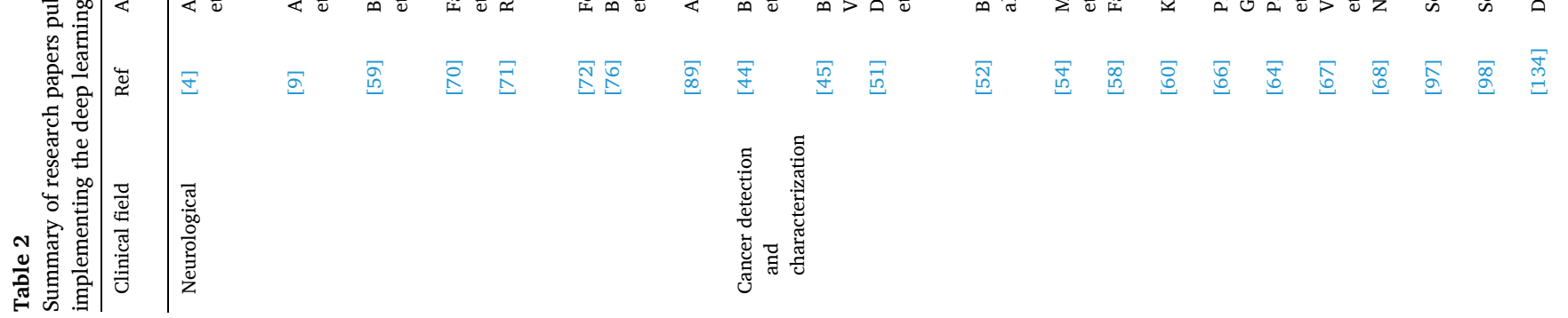




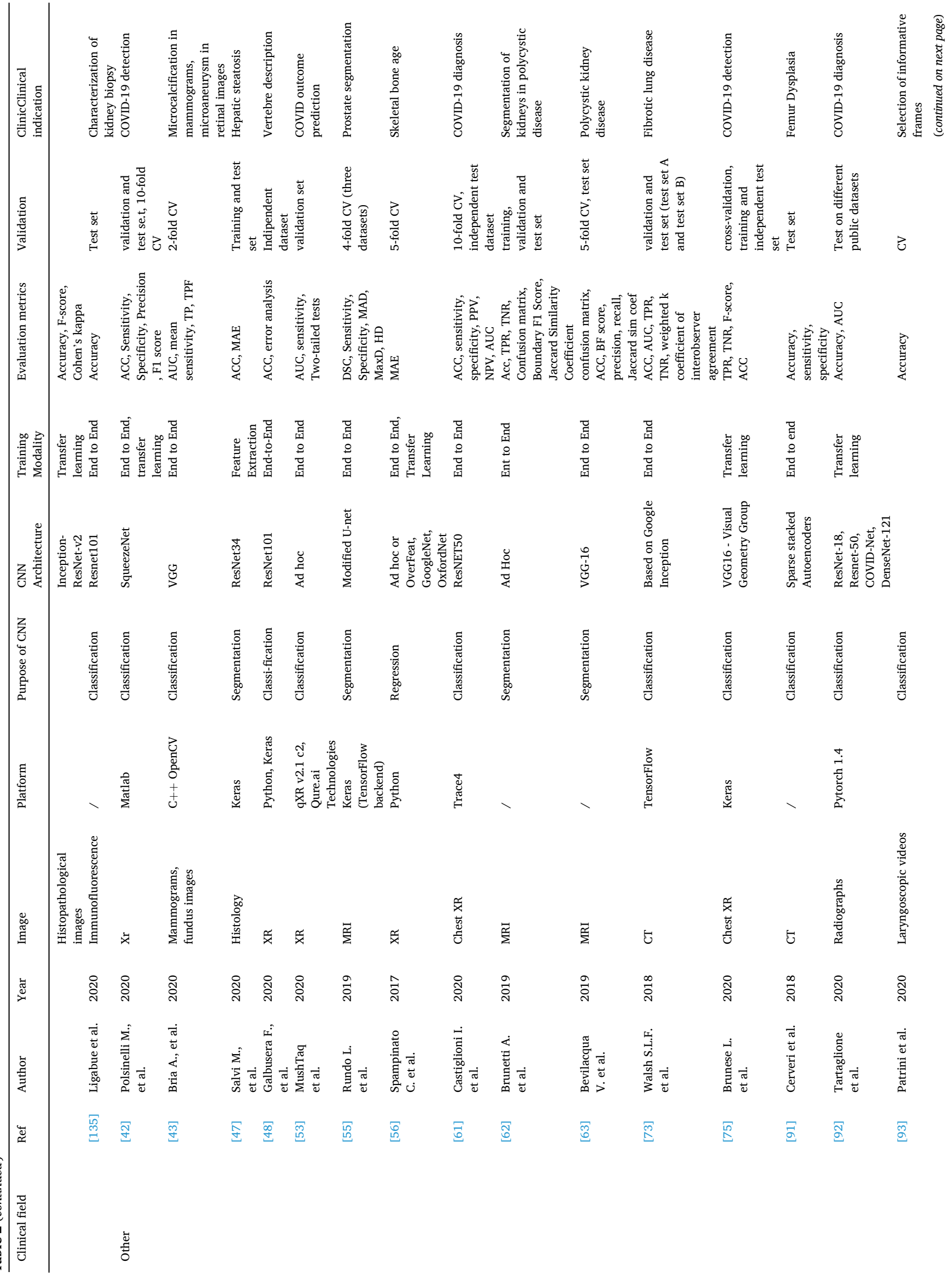




\begin{tabular}{|c|c|c|c|c|c|c|c|c|c|c|c|c|c|c|c|c|c|c|c|}
\hline 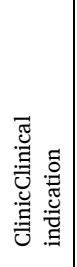 & 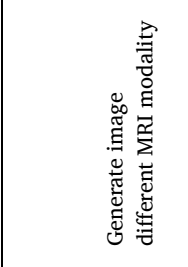 & 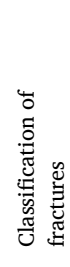 & 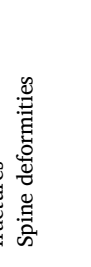 & 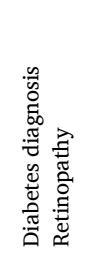 & 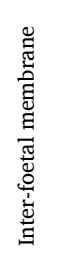 & 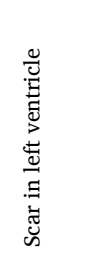 & 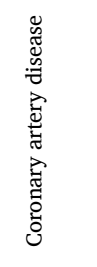 & 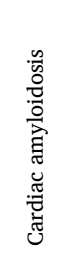 & 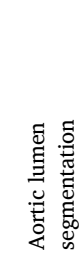 & 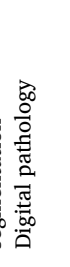 & 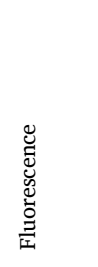 & 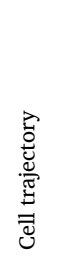 & 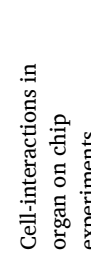 & 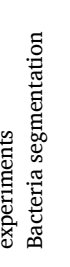 & \multicolumn{3}{|c|}{ 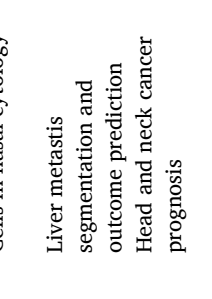 } & & 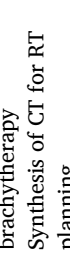 \\
\hline & 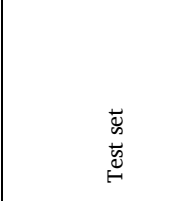 & 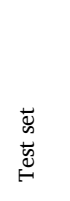 & 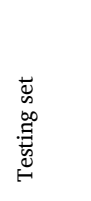 & 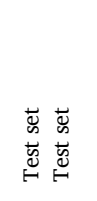 & 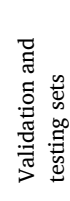 & 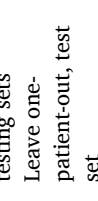 & 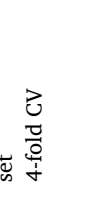 & 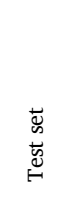 & 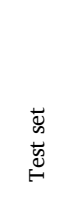 & 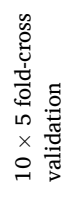 & & 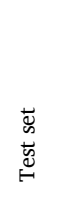 & - & & 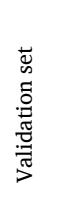 & 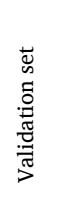 & 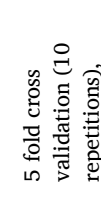 & $\begin{array}{l}\vec{z} \\
0 \\
0 \\
\frac{0}{9} \\
y\end{array}$ & 总 \\
\hline & 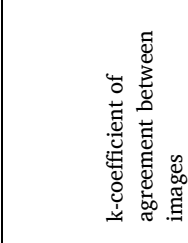 & 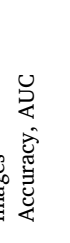 & 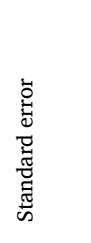 & 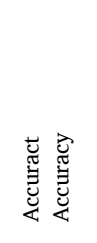 & u & 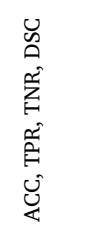 & 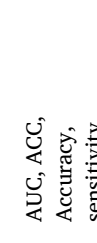 & & ù & 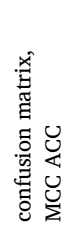 & 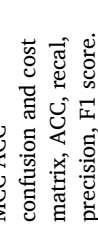 & ن & 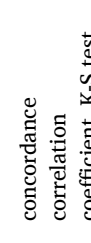 & & 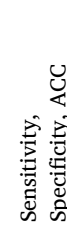 & 要 & 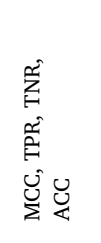 & 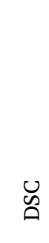 & 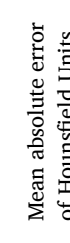 \\
\hline 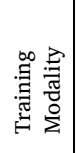 & 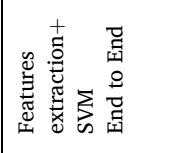 & 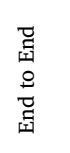 & 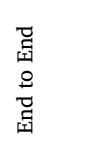 & 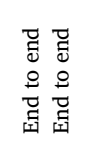 & 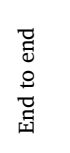 & 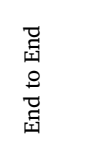 & 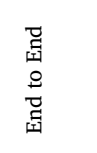 & 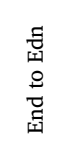 & $\begin{array}{l}\overrightarrow{7} \\
\overrightarrow{9} \\
\stackrel{9}{+} \\
\vec{\square}\end{array}$ & & 总 & 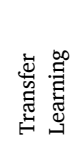 & 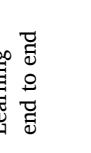 & 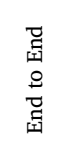 & 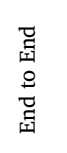 & 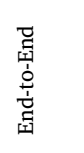 & 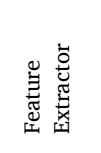 & 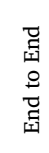 & 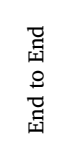 \\
\hline 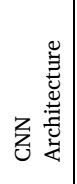 & 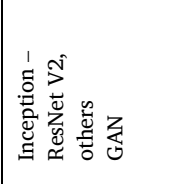 & 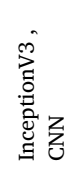 & : & 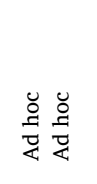 & 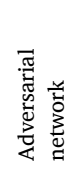 & 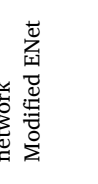 & 若 & 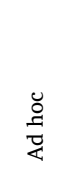 & 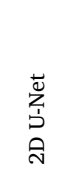 & 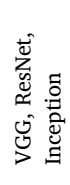 & 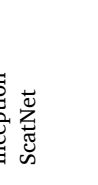 & 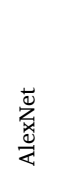 & 总 & 否 & $\begin{array}{l}\ddot{\circ} \\
\stackrel{8}{\&}\end{array}$ & 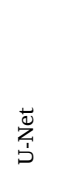 & $\frac{\mathscr{0}}{\mathscr{8}}$ & & 奀 \\
\hline & 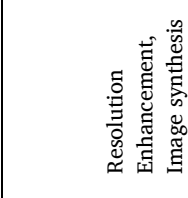 & 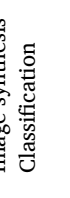 & 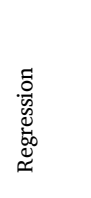 & 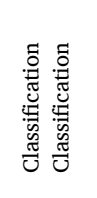 & 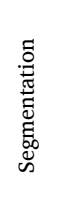 & 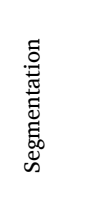 & 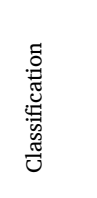 & 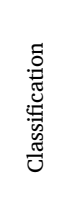 & 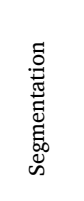 & 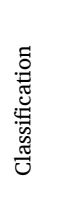 & 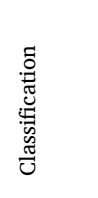 & 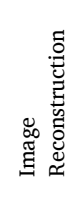 & 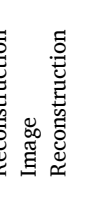 & 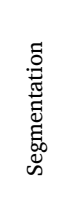 & 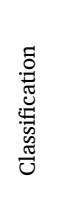 & 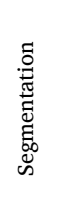 & 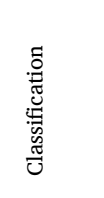 & 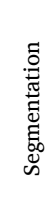 & 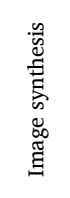 \\
\hline & 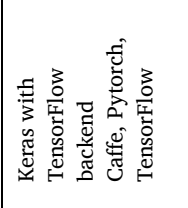 & 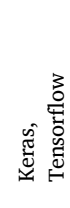 & 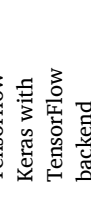 & 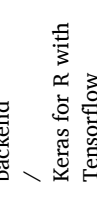 & 要 & 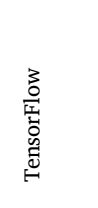 & 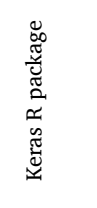 & 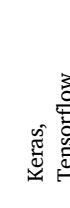 & 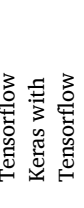 & 章 & $\lambda$ & 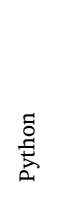 & - & 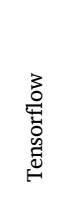 & 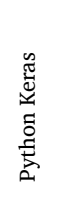 & 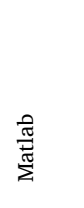 & 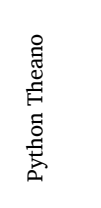 & & $\stackrel{\mathscr{\Xi}}{\mathscr{\Xi}}$ \\
\hline 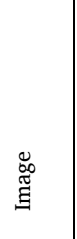 & $\overrightarrow{\tilde{s}}$ & 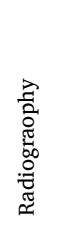 & 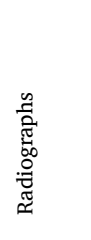 & 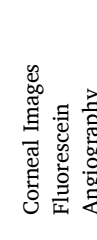 & 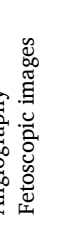 & $\begin{array}{l}\overrightarrow{\tilde{s}} \\
\underset{1}{1} \\
\text { 떱 }\end{array}$ & 司 & $\overrightarrow{\tilde{s}}$ & 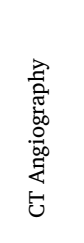 & 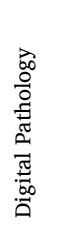 & 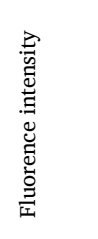 & 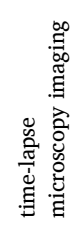 & 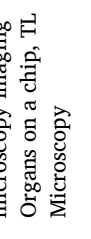 & 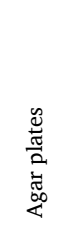 & 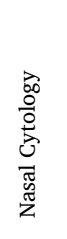 & $\forall$ & 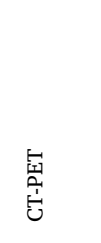 & $\overrightarrow{\tilde{z}}$ & $\overrightarrow{\tilde{\Sigma}}$ \\
\hline 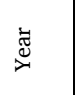 & $\stackrel{\infty}{\stackrel{\sim}{\sim}}$ & ণั & $\overrightarrow{\stackrel{े}{े}}$ & 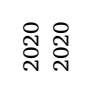 & ثें & స్ & ণั๊ & ণิి & రิ & $\stackrel{\vec{े}}{\text { वे }}$ & 亏ें & ๙ั๊ & ণิ & ণั & 亏ें & ণิ & $\stackrel{\text { 亏े }}{ }$ & ते & ลิ \\
\hline : & 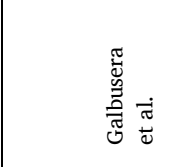 & 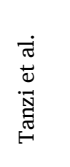 & 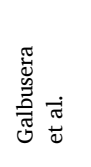 & 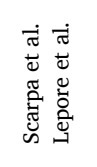 & 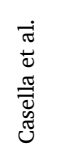 & 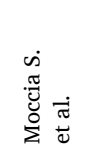 & 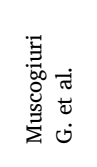 & 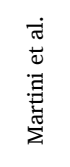 & 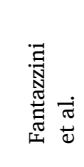 & 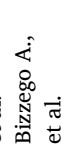 & 这 & 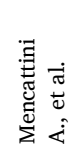 & 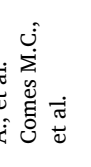 & 节 & 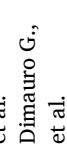 & 离 & 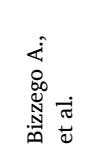 & $\begin{array}{l}\text { 元 } \\
\text { ప } \\
0 \\
\text { 胥 } \\
\text { N }\end{array}$ & 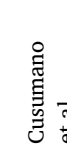 \\
\hline & $\underset{\vec{J}}{\stackrel{\Xi}{J}}$ & 趙 & $\stackrel{\infty}{\stackrel{\infty}{g}}$ & 家宫 & 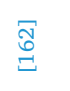 & $\underset{\Xi}{\Xi}$ & 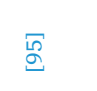 & $\underset{g}{\stackrel{g}{\Xi}}$ & 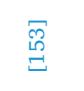 & 导 & $\bar{g}$ & 宮 & $E$ & $\bar{\Phi}$ & F & て્ఝ & $\stackrel{\Xi}{\stackrel{\Xi}{\varrho}}$ & $\check{\sigma}$ & ब్త్ర \\
\hline & & & & & & 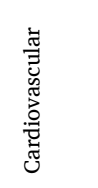 & & & & 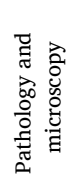 & & & & & & 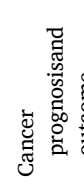 & : & & \\
\hline
\end{tabular}


$[3,4]$. The combination of four ML models based on MRI turned out to over perform each of them [23]. DTI was inquired as an input for AD patient classification either using DTI metrics (fractional anisotropy and mean diffusivity) as input for machine learning models [114] or tractography algorithms for the characterization of structural connection alterations in the brain due to $\mathrm{AD}$ [3]. A ML framework could quantify the strength of association between DTI connectivity and cognitive spectrum in Alzheimer's disease summarized by a cognitive score on a continuum range of values[122]. PET imaging combined with a SVM model can characterize hypometabolism typical of patients with $\mathrm{AD}$ [123].

Other applications to neurodegenerative disease are: diagnosis of systemic sclerosis based on texture analysis in computed tomography (CT) imaging [124] and diagnosis of multiple sclerosis by comparing different ML algorithms based on 3D T1-MRI and rsfMRI [125], where the approach based on RF feature selection and SVM provided the most accurate result. DL can be used to reconstruct MRI images from the kspace MRI of different sequences [126] or generate MRI which combine information from different modalities [70].

In the field of mental disorders AI techniques have been recently employed in the diagnosis and classification of Autism Spectrum Disorders (ASD): in [127] a SVM classification approach based on rsfMRI data was used to separate individuals with ASD from matched typical development controls. In [28] a common pattern of structural features extracted from 3D-T1 MRI was found to characterize a population of ASD individuals by means of the One-Class Classification method. SVM was applied to MRI intensity values to test sex-related structural differences of young children with ASD[18].

Concerning schizophrenia, a framework based on multiple descriptors derived from BOLD signals in fMRI during a work memory task was employed for measuring brain activity [128] and for the binary classification of patients and healthy controls [48]. For the differential diagnosis of bipolar and unipolar depression, a multiple kernel learning approach was implemented combining voxel based analysis on 3D-T1 MRI and DTI [69]. In healthy subjects, the cerebral cortex has been characterized using fractal dimension [129].

\section{Cancer diagnosis and characterization}

AI technologies are often employed for helping in detection of cancer [130-136] potentially reducing the healthcare costs due to misdiagnosis and aiding the transition towards novel precision medicine protocols. Also, AI can be used to characterize cancer by describing tumor gene mutation status $[14,24,103,133,137]$ or infiltration of nearby structures $[138,139]$.

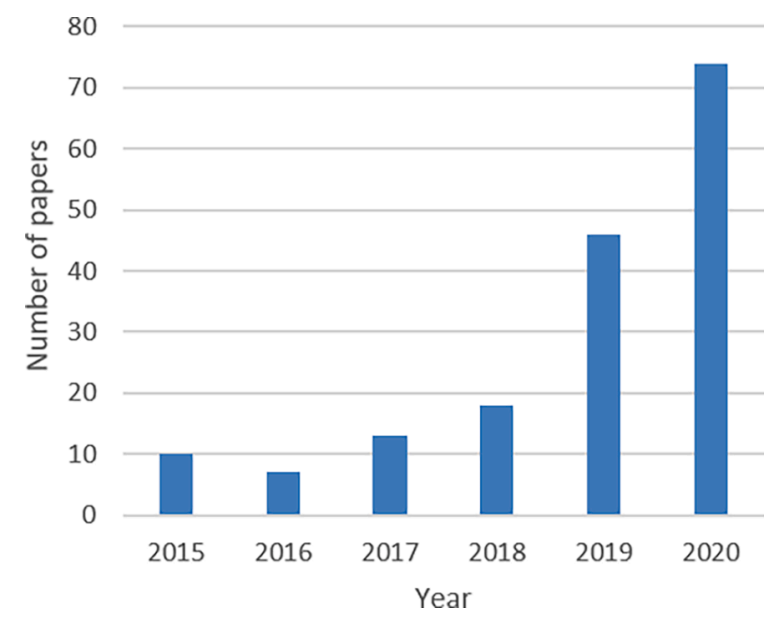

Fig. 2. histogram of studies on AI applied to imaging published in Italy included in the systematic review versus years of publication.
Microcalcifications, potentially an early sign of breast cancer, can be detected from mammograms using ML clustering methods such as kmeans $[31,34]$ or DL, which also allows segmentation of microcalcifications [43,67] and breast parenchyma [66].

Models for discriminating malignant lesions in digital mammograms which make use of RF binary classifiers have been proposed [99]. The development of deep CNN (DCNN) models has been shown to improve the diagnostic accuracy in discriminating malignant breast cancer lesions in mammography. A DCNN architecture was proposed for reducing false negatives while still keeping acceptable accuracy, showing that random initialization $\mathrm{CNN}$ architecture can provide practical aid in the classification and staging of breast cancer [51].

Recently, AI was applied to a novel instrumentation for diagnosis of breast cancer, Contrast-enhanced spectral mammography (CESM), where dual-energy mammograms are acquired after contrast medium administration. In this way it can provide also images where the only contrast medium is visible. Textural features extracted from CESM could discriminate benign and malignant breast lesions using SVM classifier [68] and a fully automatic system as a diagnostic support tool for the clinicians using a RF classifier outperformed the human reader [15]. ML and DL can also be used in tomosynthesis for distinguishing malignant lesions [45].

By integrating genomic data of the tumor such as MicroRNAs with MRI radiomic features, breast cancer can be classified as belonging to subtype Luminal A, Luminal B, HER2+, or Basal using SVM [85].

Breast biopsy, normally done under US image guidance to direct the needle towards the target, is the preferred technique to evaluate the malignancy of screening detected breast lesions. A U-Net architecture was trained to predict probe-induced deformations on the breast anatomy was trained in a realistic multi-modality breast phantom (Model 073; CIRS, Norfolk, VA, USA) with 10 inner lesions of diameter of 5-10 $\mathrm{mm}$ in order to provide real-time position of breast lesions [54].

Prostate lesions could be characterized as cancer by extracting radiomic features from $\mathrm{T} 2 \mathrm{w}$ and diffusion weighted imaging (DWI) and then using a classifier based on discriminant analysis (DA) and generalized linear model[140]. Gleason score [141] and extracapsular extension [138] are also associated with MRI textural features.

Brain cancer diagnosis, mostly based on MRI images, remains a challenging, error-prone and highly specialized task. An ensemble learner based on a set of radiomic features to detect and grade brain cancers was tested on more 111,000 brain MRI exams belonging to two public data-sets [30]. Radiomic features extracted from dynamic susceptibility contrast maps were used as input for $\mathrm{ML}$ to predict $\mathrm{O}^{6}$ methylguanine-DNA methyltransferase (MGMT) gene promoter, a predictor of response of glioblastoma multiforme to temozolomide, and a prognostic indicator of survival time [24]. Deep CNN were applied to 3D T1-MRI and DWI maps in a retrospective study for the classifications of meningiomas, turning out that the discrimination from apparent diffusion coefficient maps has the largest area under the ROC curve [44].

Achieving accurate and reproducible tumor volume segmentation is still a challenge in neuro-radiosurgery. Computer-assisted approaches such as NeXt [142] for necrosis extraction, and MedGA [143] for brain metastatic cancer segmentation in neuro-radiosurgery [35]. Automatic strategies for the segmentation of brain metastases on 11C-labeled Methionine PET imaging were developed [52,144].

Low-dose CT screening allows early detection of lung cancer and subsequent mortality reduction, but it's affected by frequent false positives, inter/intra observer variation and uncertain diagnoses of lung nodules. AI approaches for lung cancer cancer detection usually involve radiomic features extraction and selection, then building a ML model such as ANN [145]. ML showed a good accuracy in distinguishing benign from malignant nodules in low-dose CT screening, and outperformed the clinical standard in an independent validation cohort [10]. A lung cancer detection model using RF and ANN for radiomic features selection was developed for chest digital tomosynthesis, [27], and the least absolute shrinkage and selection operator (LASSO) logistic 
(a)

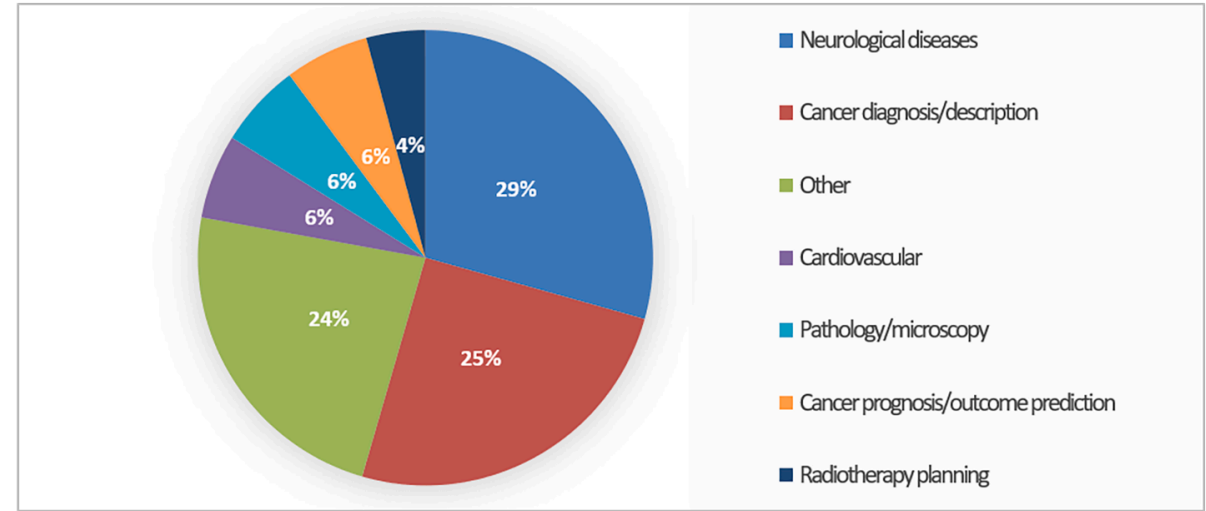

(b)

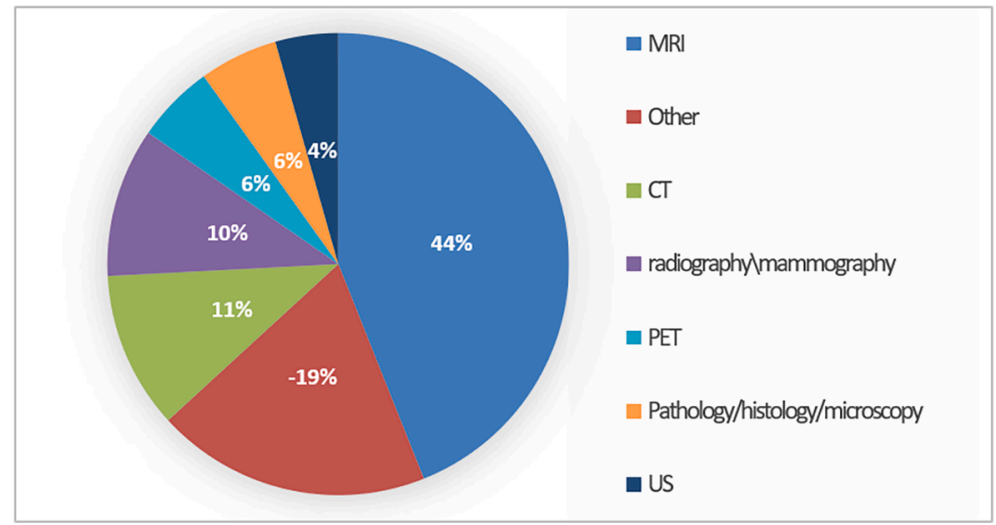

(c)

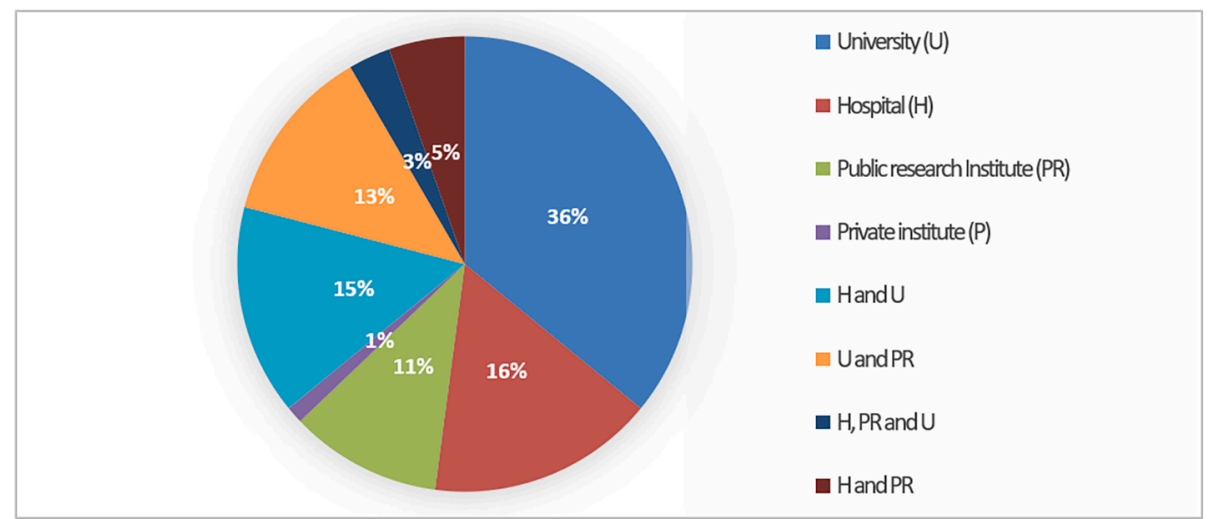

Fig. 3. Pie charts showing the distribution of clinical indication (a), imaging technique (b) and type of institution (c) for studies on AI applied to imaging in Italy.

regression modelwas used to predict positive lymph nodes [11]. DL was also applied with promising results to multimodality PET/CT lung images for the classification of lung cancer lesions as T1-T2 or T3-T4 [60].

Another field where AI can play an important role is dermatology, where Multiple Instance Learning was successfully investigated for diagnosing melanoma from common nevi in dermoscopic color images [146]. Other cancer types which have been investigated using ML or DL for detection or characterization include bone chondrosarcoma[81], head and neck [147], and renal cell carcinoma [148].

\section{Cardiovascular}

ML tools can aid cardiologists in early diagnosis of cardiovascular diseases such as amyloidosis[149] using a variety of imaging techniques. Coronary arterial disease can be diagnosed by application of SVM to trajectories of landmarks in echogardiography [150], and ventricular failure by integration of these with biochemical and clinical data using Naïve Bayes ML [151].
Predictive models of coronary health disease, cardiovascular disease and chronic heart failure integrating parameters obtained by radiodensitometric CT images of skeletal muscle were proposed, where RFs algorithm yielded the highest classification performance [83]. In myocardial perfusion SPECT imaging, small vessel disease [8] and coronary arterial disease [152] could be classified by applying ML to imaging features. A novel DL network to classify coronary computed tomography angiography (CCTA) in the correct Coronary Artery Disease Reporting and Data System (CAD-RADS) category showed the ability to differentiate with high diagnostic accuracy patients with CADRADS 0 (patients do not appear to derive benefit from medical therapy) and CADRADS greater than 0 (patients appear to derive benefit from medical therapy) [95].

U-net, one of the most popular DL architectures for image segmentation, was studied for segmenting aortic lumen in angiography [153], while left ventricle scar tissue was segmented in cardiac MRI with late gadolinium enhancement (CMR-LGE) images based on modified ENet (Efficient Neural Network) [74]. Cardiac substructures can be 
segmented using hierarchical clustering [154].

\section{Other diagnostic applications}

Prostate zonal segmentation is a time-expensive and operatordependent procedure when performed manually. Two DL networks, USE-Net and Enc-Dec USE-Net, obtained by modifying U-Net, one of the most effective CNNs in biomedical image segmentation, were tested on three T2-weighted MRI datasets by different institutions [55]. An automatic segmentation method applied to multispectral MRI could segment the prostate gland effectively based on an unsupervised ML, the Fuzzy C-Means (FCM) clustering [36]. Other applications of DL for automated identification of disease include investigating fibrotic lung disease segmentation in high-resolution CT [73].

Almost immediately after the outbreak of the recent COVID-19 pandemic disease in Italy, it was clear that DL, by automatically assessing image features possibly correlated with COVID-19 from chest radiography (Fig. 4), can be a valuable tool for diagnosis of COVID from pneumonia or healthy patient $[61,75]$. Fig. 4 shows chest radiographies overlayed with class activation maps (CAM), the regions considered relevant by a CNN to classify the image.

Among the different architectures investigated, an ensemble of 10 ResNET50 CNNs showed AUC equal to 0.81 in the independent test set [61]. A previously trained VGG-16 was fine-tuned for lung disease detection and classification (pneumonia or COVID-19) with accuracies in the test set equal to 0.96 and 0.98 , respectively [75]. A commercial system based on ResNets showed comparable performance to radiologist-assessed score on chest X-ray [53].

CT was also considered for automated diagnosis of COVID-19 using DL, by using a modified version of SqueezeNet CNN, achieving a slightly higher accuracy $(85.0 \%)$ than the original SqueezeNet model while maintaining its computational efficiency [42]. AI models were also built for short-term outcome prediction of COVID-19 patients, i.e. favorable vs mechanical ventilation/intensive care or death, and CT features combined with clinical data in a SVM classifier achieved AUC of 0.92 [155].

AI has proven to be a promising tool for assessing bone mineral density, trabecular bone score and bone strain from dual x-ray absorptiometry. In a retrospective study of 125 postmenopausal women, bioochemical markers of bone turnover (type I collagen carboxy-terminal telopeptide, alkaline phosphatase, vitamin D) were measured and correlated with fragility fractures by using a neural network analysis with the auto contractive map algorithm [156]. On radiographies, DL can detect fractures [157] and spine deformities [158].

The assessment of the skeletal bone age by left-hand radiography is a common practice to investigate endocrinology, genetic and growth disorders in children. Three different CNN, with similar architecture showed an average error of estimated age of 0.8 years on a public dataset of 1391 subjects, covering all age ranges, genders and races [45].

To determine the 3D position and orientation of vertebras from planar radiographs, a modified Resnet was trained on synthetic radiographies derived by projection from CT, resulting in errors lower than $3^{\circ}$ in $86 \%$ of the test dataset [48].

Diabetes-related neuropathy could be diagnosed by CNN from confocal microscopy [159] and retinopathy from fluoresceing angiography images [160].

\section{Pathology and microscopy}

Pathological sciences offer another foreground application for AI in imaging. In stained histopathologic images of breast tissue, DL can automatically detect neoplastic epithelium, which is made difficult by extreme variability of its morphology [25].

Nasal cytology, by allowing non-invasive study of nasal mucosa cells, is increasingly important in otorhinolaryngology. DL has been proposed for classification of cells (e.g. Neutrophils, Lymphocytic) in rhino- cytogram microscopic images [94]. Type 1 diabetes mellitus can be diagnosed from Laudan intensity images of red blood cells obtained with confocal microscope, using ML classifier and radiomic features [161].

Clonogenic assays, quantifying the anti-proliferative effect of treatments on cell cultures, are usually performed by error-prone and operator-dependent manual counting of cells. Besides, conventional assessment does not deal with the colony size, which is generally correlated with the delivered radiation dose or administered cytotoxic agent. MF2C3 computational method leverages spatial Fuzzy C-Means clustering on multiple local features (i.e., entropy and standard deviation extracted from the input color images acquired by a generalpurpose flat-bed scanner) for quantification of area, count and and size of the colony, along with the growth rate [38].

Multi-scale Generative Adversarial Network (GAN) was used to produce high quality inter-leaved video frames in videos of time-lapse microscopy for assessing biological processes, such as cell migrations and interactions [77].

Semantic segmentation of kidneys in MRI in Autosomal Dominant Polycystic Kidney Disease, an hereditary disease which changes kidney appearance, was achieved using modified VGG-16 to detect and segment the region of interest [63].

Fetal biometrics on ultrasound imaging, in particular measurement of fetal head thickness, is prone to inter-user variability. By using two DL networks, a pretrained and fine-tuned tiny-YOLOv2 for localizing the fetal head and U-net for performing regression of fetal head thickness, it is possible to accomplish this task automatically and reproducibly [57]. DL was applied to foetoscopic images for segmentation of structures [162]. ML applied to MRI image features can identify placenta accrete spectrum, trophoblasts' abnormal invasion into the myometrial layer [82].

\section{Cancer prognosis and therapy outcome prediction}

Recently, quantitative analysis of the tumor phenotype in radiological images by extraction of a large number of radiomic features have been coupled with ML classifiers with the aim of producing prognostic and predictive models where PET images, by providing tumor metabolism information, can play a key role. A ML approach was used to predict disease progression in high risk prostate cancer who underwent restaging 18F-Cho PET/CT by employing neighborhood component analysis (NCA) followed by discriminant analysis (DA) ML [5]. Radiomic features extracted from brain metastases in 11C-MET PET, reduced by correlation matrix analysis and point-biserial correlation coefficient, were included in a ML classifier for responders/ non-responder patients after stereotactic radiotherapy [144].

A ML pipeline, based on RF applied to radiomic features extracted from the semi-automatically segmented tumor on MRI, was proposed to predict volume change of acoustic neuroma following Cyberknife radiosurgery [26].

Different ensemble ML using CT radiomics features were investigated for prediction of overall survival in patients with Non-Small Cell Lung Cancer [11]. Interestingly, the accuracy improved when using the clinical target volume, confirming the hypothesis that, in the surroundings of the visible tumor, there is useful information to predict patients' outcome [6]. A retrospective study recently started in the framework of Artificial Intelligence in Medicine (AIM), a project founded by INFN, aims to investigate imaging and dose biomarkers for clinical outcome of pediatric patients affected by medulloblastoma who underwent Cranio-Spinal Irradiation (CSI) using Helical Tomotherapy [163].

Radiomics based ML models can also be studied to predict side effects or radiotherapy, such as late radiation induced fibrosis. For this purpose, the HU in CT images and the dose distribution were converted in 3D Relative Electron Density (3D-RED) and 3d Biologically Effective Dose (3D-BED). The best model for predicting late fibrosis was a 7 variables SVM, according to its accuracy in a 5-fold cross validation [7]. 
Likelihood-Fuzzy Analysis (LFA) models were trained on a dataset of 37 IMRT patients to classify patients at risk of parotid gland shrinkage and long-term xerostomia and, combining clinical, dosimetric and with radiomics features from $\mathrm{CT}$ images acquired before, at the middle and after RT, to obtain accurate models with high performance [164]. Prediction of response to chemo-RT for anal and rectal cancer using ML and radiomics is also an active area of investigation $[28,165]$.

The use of DL models, which can be trained to predict patient outcome from images, is still at its beginning. DL has been investigated for predicting recurrence of Head and neck cancer from CT/PET [166] as well as for predicting response of liver metastases to chemotherapy [65].

(a)

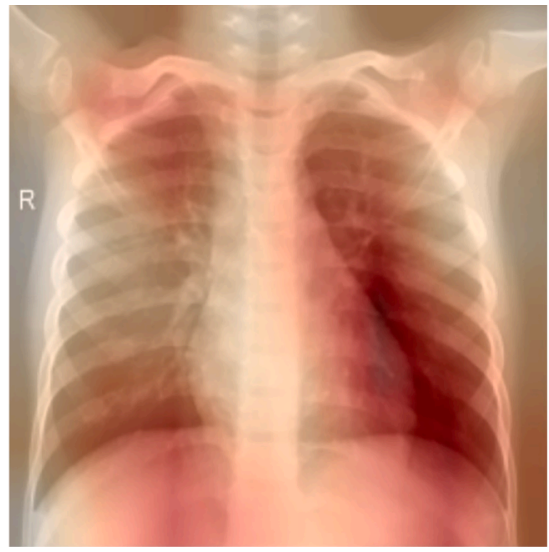

(c)

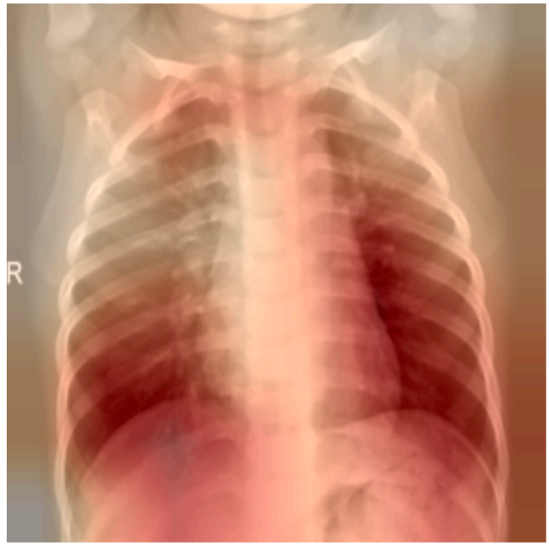

(e)

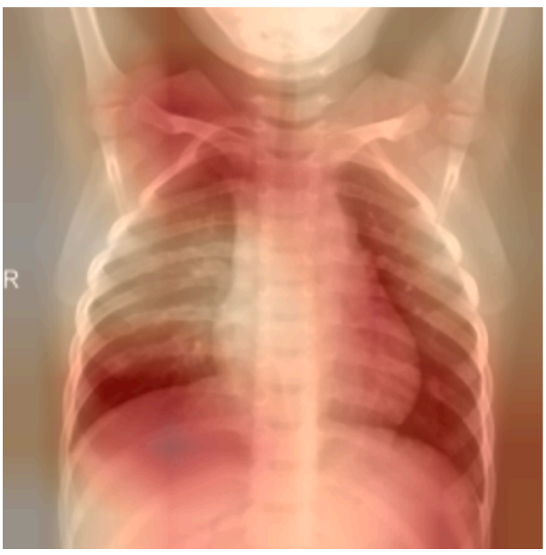

\section{Radiotherapy}

Use of AI in radiotherapy include segmentation of structures used for planning of the treatment, such as cathethers in brachytherapy, [96]. A co-segmentation method to integrate the segmented Biological Target Volume (BTV), using $\left[{ }^{11} \mathrm{C}\right]$-Methionine-PET images, and the segmented Gross Target Volume (GTV), on the respective co-registered MR images, was proposed [37].

Tumor motion increases uncertainty of radiotherapy delivery. A prediction model was implemented to predict tumor motion trajectories from cineMRI (4D-MRI) acquired with $3 \mathrm{~T}$ scanner, which involved SVM applied to anatomy landmarks positions [167]. A custom ML algorithm was developed to outline prostate on US registered with pretreatment CT to automatically determine target displacement during RT [168].

(b)

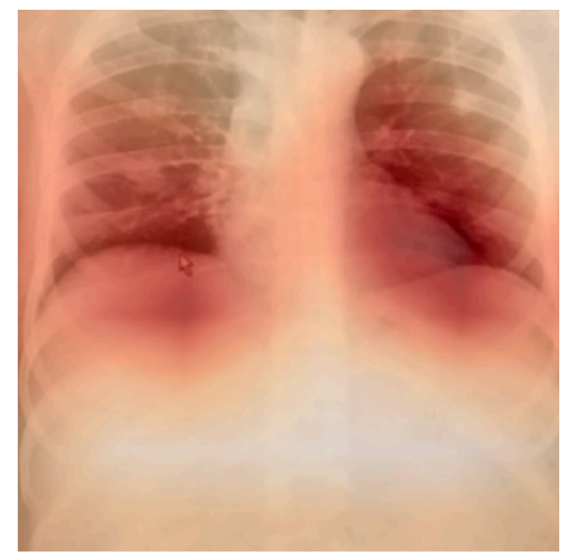

(d)

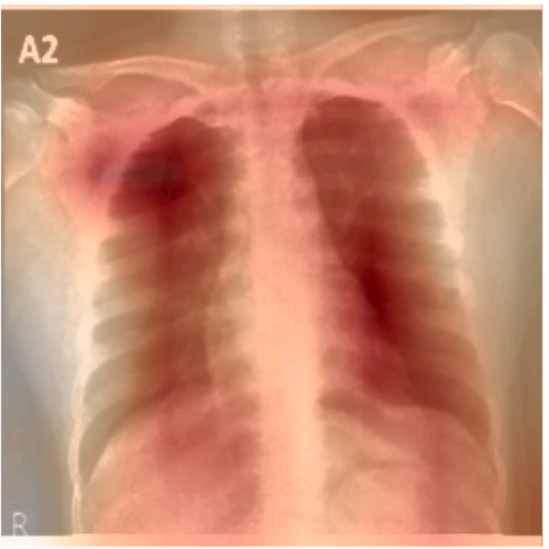

(f)

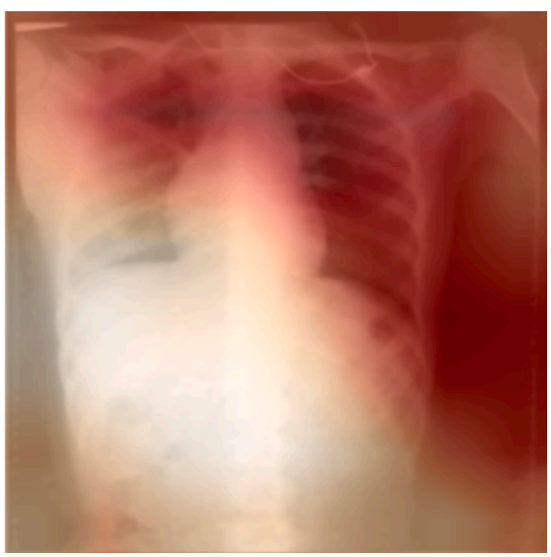

Fig. 4. Examples of activation maps calculated from chest radiographies of patients with Keras, negative (a, c and e) and positive (b, d and f) for COVID related pneumonia. 
Synthetic CT generation by DL from MRI used for image-guidance in MRI-LINAC allows for daily dose calculation [169].

Using cone-beam CT or MV-CT used for radiotherapy image guidance, ML can identify significant changes of patient anatomy during therapy and predict patients who would benefit from adaptive radiotherapy [170-172].

\section{AI in Italy: Challenges and promises}

Among the limitations of this review is that AI is a rapidly evolving field and providing a thorough report on its status may be a difficult task. Some studies may have been missed by the Pubmed search, in particular those which mentioned only specific AI methods ("decision trees"). On the other hand, studies provided by members of the working group may be affected by biases according to the background and current activity of researchers. Notwithstanding these limitations, this review, the first of this kind to the best of our knowledge, provides a snapshot of $\mathrm{AI}$ in imaging in Italy, and shows its rapid uprise. This confirms how many researchers, including medical physicists, are enthusiastic to apply AI in the healthcare field. However, there are still many open challenges that need to be confronted in order to untap the potential of this research field.

\section{Challenges}

Compared with ML, a lower number of studies among those identified (35\%) used DL. Although DL techniques might achieve remarkable performance, they are characterized by the high complexity of configuration, need for a large amount of images for training, long training times and a performing computing infrastructure based on advanced hardware (e.g., GPU, such as Titan XP (NVIDIA, Santa Clara, CA) graphic processing units, a Xeon E5-2640 v4 $2.1 \mathrm{GHz}$ (Intel, Santa Clara, CA) processor, and $32 \mathrm{~GB}$ of random access memory in [44] cluster or cloud computing). This could explain the lesser diffusion of these techniques, and common efforts should be focused on obtaining cloud computing resources to be shared among the MP community for research on AI.

ML algorithms are very flexible and adaptable to the learning dataset and, thus, prone to overfit, which results in not generalizable models. In order to avoid overfitting and validate models, ML and DL require huge datasets. The large majority of the studies identified are single center studies used internal validation techniques, as 10 -fold cross-validation or leave-one-out, which do not estimate model capability to perform well on unseen samples. Only a minority of studies aimed at external validation $[55,145]$ or were multicentric e.g. $[170,173]$.

Another issue is about data: because of privacy/ownership concerns, researchers cannot share data easily. For these reasons, it would be desirable to have common data-centers and large databases of digital medical images and annotated information, which need computation and data sharing infrastructures.

The image acquisition procedures used in different institutions are often not comparable, and the parameters extracted show a dependence related to scanners of different vendors. Also, DL networks depend on the learning techniques and data used and artefacts can introduce unexpected issues in the algorithm.

Model transparency, meaning that its formulas and code should be available and comprehensible is considered fundamental for translation of AI into clinical practice [174]. However, not all of the studies clearly reported the software or platform used for AI or the architecture for DL. The research community should be transparent on how a model was obtained, also because this helps the user to understand its applicability in a clinical setting.

There is need for common guidelines and protocols on how to perform and report AI, ML and DL, in the spirit of the TRIPOD [175], where criteria are given for development, transparent reporting and validation of models. These should cover every step of the AI framework, including the choice of proper ML algorithms and architecture, in order to achieve standardization and increase quality and transparency of models and data. Moreover, they should deal with legal and ethical issues, in the context of Italian and European regulation on AI [174].

\section{Promises of AI in Italy}

AIFM, is active, also through its working group on AI, AI4FM, in increasing the level of collaboration with other scientific and professional organizations in healthcare involved in application of AI [176].

The Artificial Intelligence in Medicine (AIM) experiment [177] aims to exploit the expertise of INFN and associated researchers on medical data processing and enhancement, and turn it in the development of advanced and effective analysis instruments to be eventually clinically validated and translated into products. A network of fruitful interactions between INFN Physicists and Clinicians of several Italian hospitals and clinical research centers has been built in the last two decades, also due to specific research initiatives funded by INFN-CSN5 (National Scientific Committee 5).

Large data sharing scientific initiatives, such as Alzheimer Disease Neuroimaging Initiative - ADNI [123] and Autism Brain Imaging Data Exchange - ABIDE [16], have also promoted the investigation of new methods based on AI algorithms in different clinical settings. A multicentric validation was successfully performed on a SVM method to diagnose amyotrophic lateral sclerosis from PET [178]. In a similar spirit, a large international consortium performed validation of ML for pigmented skin lesion classification by Comparing them against human readers[179].

In 2016 an Italian network project has started with a triple aim: optimize, harmonize and share advanced MR imaging protocols in the neurological field [180]. This approach helps to minimize scanner heterogeneity issues and improves data aggregation. The creation in such contexts of databases integrating clinical and radiological information would represent a solid basis for the application of ML techniques.

\section{Conclusions}

The present work shows that we are witnessing an unprecedented interest in AI applied to imaging in Italy, in a diversity of fields and imaging techniques. Further initiatives are needed to build common frameworks, collaborations among different types of institutions, and guidelines for research on AI and its safe deployment in healthcare.

\section{Reference}

[1] Associazione Italiana di Fisica Medica (AIFM). AIFM n.d. https://www. fisicamedica.it/en/ (accessed March 1, 2021).

[2] Associazione Italiana di Fisica Medica e Sanitaria. Big Data e Intelligenza Artificiale: il gruppo di lavoro AIFM. AIFM 2020. https://www.fisicamedica.it/itemi-della-fisica-medica/big-data-e-intelligenza-artificiale/big-data-eintelligenza-artificiale-il-gruppo-di-lavoro-aifm/ (accessed March 1, 2021).

[3] Lella E, Lombardi A, Amoroso N, Diacono D, Maggipinto T, Monaco A, et al. Machine learning and DWI brain communicability networks for Alzheimer's disease detection. Appl Sci 2020;10. https://doi.org/10.3390/app10030934.

[4] Amoroso N, Diacono D, Fanizzi A, La Rocca M, Monaco A, Lombardi A, et al. Deep learning reveals Alzheimer's disease onset in MCI subjects: results from an international challenge. Mach Learn Neuroimaging Chall Autom Diagn Alzheimer's Dis 2018;302:3-9. https://doi.org/10.1016/j. jneumeth.2017.12.011.

[5] Alongi P, Laudicella R, Stefano A, Caobelli F, Comelli A, Vento A, et al. Choline $\mathrm{PET} / \mathrm{CT}$ features to predict survival outcome in high risk prostate cancer restaging: a preliminary machine-learning radiomics study. Q J Nucl Med Mol Imaging Off Publ Ital Assoc Nucl Med AIMN Int Assoc Radiopharmacol IAR Sect Soc Of 2020. https://doi.org/10.23736/S1824-4785.20.03227-6.

[6] D'Amico NC, Sicilia R, Cordelli E, Tronchin L, Greco C, Fiore M, et al. Radiomicsbased prediction of overall survival in lung cancer using different volumes-ofinterest. Appl Sci 2020;10. https://doi.org/10.3390/app10186425.

[7] Avanzo M, Pirrone G, Vinante L, Caroli A, Stancanello J, Drigo A, et al. Electron density and biologically effective dose (BED) radiomics-based machine learning models to predict late radiation-induced subcutaneous fibrosis. Front Oncol 2020; 10:490. https://doi.org/10.3389/fonc.2020.00490.

[8] Ricciardi C, Cantoni V, Improta G, Iuppariello L, Latessa I, Cesarelli M, et al. Application of data mining in a cohort of Italian subjects undergoing myocardial 
perfusion imaging at an academic medical center. Comput Methods Programs Biomed 2020;189:105343. https://doi.org/10.1016/j.cmpb.2020.105343.

[9] Amoroso N, La Rocca M, Bellantuono L, Diacono D, Fanizzi A, Lella E, et al. Deep learning and multiplex networks for accurate modeling of brain age. Front Aging Neurosci 2019;11:115. https://doi.org/10.3389/fnagi.2019.00115.

[10] Garau N, Paganelli C, Summers P, Choi W, Alam S, Lu W, et al. External validation of radiomics-based predictive models in low-dose CT screening for early lung cancer diagnosis. Med Phys 2020;47:4125-36. https://doi.org/ $10.1002 / \mathrm{mp} .14308$.

[11] Botta F, Raimondi S, Rinaldi L, Bellerba F, Corso F, Bagnardi V, et al. Association of a CT-based clinical and radiomics score of non-small cell lung cancer (NSCLC) with lymph node status and overall survival. Cancers 2020;12. https://doi.org/ 10.3390/cancers12061432.

[12] Pantoni L, Marzi C, Poggesi A, Giorgio A, De Stefano N, Mascalchi M, et al. Fracta dimension of cerebral white matter: A consistent feature for prediction of the cognitive performance in patients with small vessel disease and mild cognitive impairment. NeuroImage Clin 2019;24:101990. https://doi.org/10.1016/j. nicl.2019.101990.

[13] Nero C, Ciccarone F, Boldrini L, Lenkowicz J, Paris I, Capoluongo ED, et al Germline BRCA 1-2 status prediction through ovarian ultrasound images radiogenomics: a hypothesis generating study (PROBE study). Sci Rep 2020;10: 16511. https://doi.org/10.1038/s41598-020-73505-2.

[14] Barricelli BR, Casiraghi E, Gliozzo J, Huber V, Leone BE, Rizzi A, et al. ki67 nuclei detection and ki67-index estimation: a novel automatic approach based on human vision modeling. BMC Bioinf 2019;20:733. https://doi.org/10.1186/ s12859-019-3285-4.

[15] Fanizzi A, Losurdo L, Basile TMA, Bellotti R, Bottigli U, Delogu P, et al. Fully automated support system for diagnosis of breast cancer in contrast-enhanced spectral mammography images. J Clin Med 2019;8:891. https://doi.org/ 10.3390/jcm8060891.

[16] Lombardi A, Amoroso N, Diacono D, Monaco A, Tangaro S, Bellotti R. Extensive evaluation of morphological statistical harmonization for brain age prediction. Brain Sci 2020;10:10.3390/brainsci10060364.

[17] Spera G, Retico A, Bosco P, Ferrari E, Palumbo L, Oliva P, et al. Evaluation of altered functional connections in male children with autism spectrum disorders on multiple-site data optimized with machine learning. Front Psychiatry 2019;10: 620.

[18] Retico A, Giuliano A, Tancredi R, Cosenza A, Apicella F, Narzisi A, et al. The effect of gender on the neuroanatomy of children with autism spectrum disorders: a support vector machine case-control study. Mol Autism 2016;7:5-015-0067-3. eCollection 2016. https://doi.org/10.1186/s13229-015-0067-3.

[19] Moccia S, Mattos LS, Patrini I, Ruperti M, Poté N, Dondero F, et al. Computerassisted liver graft steatosis assessment via learning-based texture analysis. Int $\mathrm{J}$ Comput Assist Radiol Surg 2018;13:1357-67. https://doi.org/10.1007/s11548018-1787-6.

[20] Galli M, Zoppis I, De Sio G, Chinello C, Pagni F, Magni F, et al. A support vector machine classification of thyroid bioptic specimens using MALDI-MSI data. Adv Bioinforma 2016;2016:e3791214. https://doi.org/10.1155/2016/3791214.

[21] Militello C, Vitabile S, Rundo L, Russo G, Midiri M, Gilardi MC. A fully automatic 2D segmentation method for uterine fibroid in MRgFUS treatment evaluation. Comput Biol Med 2015;62:277-92. https://doi.org/10.1016/j. compbiomed.2015.04.030.

[22] Avanzo M, Stancanello J, El Naqa I. Beyond imaging: the promise of radiomics. Phys Medica PM Int J Devoted Appl Phys Med Biol Off J Ital Assoc Biomed Phys AIFB 2017;38:122-39.

[23] Battineni G, Chintalapudi N, Amenta F, Traini E. A Comprehensive MachineLearning Model Applied to Magnetic Resonance Imaging (MRI) to Predic Alzheimer's Disease (AD) in Older Subjects. J Clin Med 2020;9:10.3390/ jcm9072146.

[24] Crisi G, Filice S. Predicting MGMT Promoter methylation of glioblastoma from dynamic susceptibility contrast perfusion: A radiomic approach. J Neuroimaging Off J Am Soc Neuroimaging 2020;30:458-62. https://doi.org/10.1111/ jon. 12724 .

[25] Salvi M, Molinari F, Dogliani N, Bosco M. Automatic discrimination of neoplastic epithelium and stromal response in breast carcinoma. Comput Biol Med 2019; 110:8-14. https://doi.org/10.1016/j.compbiomed.2019.05.009.

[26] D'Amico NC, Sicilia R, Cordelli E, Valbusa G, Grossi E, Zanetti IB, et al. Early radiomics experiences in predicting cyberknife response in acoustic neuroma. ACM SIGBioinformatics Rec 2019;8:11-3. https://doi.org/10.1145/ 3307616.3307620 .

[27] Chauvie S, De Maggi A, Baralis I, Dalmasso F, Berchialla P, Priotto R, et al. Artificial intelligence and radiomics enhance the positive predictive value of digital chest tomosynthesis for lung cancer detection within SOS clinical trial. Eu Radiol 2020;30:4134-40

[28] Ferrari R, Mancini-Terracciano C, Voena C, Rengo M, Zerunian M, Ciardiello A et al. MR-based artificial intelligence model to assess response to therapy in locally advanced rectal cancer. Eur J Radiol 2019;118:1-9. https://doi.org/ 10.1016/j.ejrad.2019.06.013.

[29] Inglese P, Amoroso N, Boccardi M, Bocchetta M, Bruno S, Chincarini A, et al. Multiple RF classifier for the hippocampus segmentation: method and validation on EADC-ADNI Harmonized Hippocampal Protocol. Phys Med 2015;31:1085-91. https://doi.org/10.1016/j.ejmp.2015.08.003.

[30] Brunese L, Mercaldo F, Reginelli A, Santone A. An ensemble learning approach for brain cancer detection exploiting radiomic features. Comput Methods Programs Biomed 2020;185:105134. https://doi.org/10.1016/j.cmpb.2019.105134.
[31] Basile TMA, Fanizzi A, Losurdo L, Bellotti R, Bottigli U, Dentamaro R, et al. Microcalcification detection in full-field digital mammograms: a fully automated computer-aided system. Phys Med 2019;64:1-9. https://doi.org/10.1016/j. ejmp.2019.05.022.

[32] Gallivanone F, Interlenghi M, Canervari C, Castiglioni I. A fully automatic, threshold-based segmentation method for the estimation of the Metabolic Tumor Volume from PET images: validation on 3D printed anthropomorphic oncological lesions. J Instrum 2016. https://doi.org/10.1088/1748-0221/11/01/C01022.

[33] Schenone D, Lai R, Cea M, Rossi F, Torri L, Bignotti B, et al. Radiomics and artificial intelligence analysis of CT data for the identification of prognostic features in multiple myeloma. Proc.SPIE, vol. 11314, 2020. https://doi.org/ $10.1117 / 12.2548983$

[34] Fanizzi A, Basile TMA, Losurdo L, Bellotti R, Bottigli U, Dentamaro R, et al. A machine learning approach on multiscale texture analysis for breast microcalcification diagnosis. BMC Bioinformatics 2020;21:91-020-3358-4. https://doi.org/10.1186/s12859-020-3358-4.

[35] Militello C, Rundo L, Vitabile S, Russo G, Pisciotta P, Marletta F, et al. Gamma Knife treatment planning: MR brain tumor segmentation and volume measurement based on unsupervised Fuzzy C-Means clustering. Int J Imaging Syst Technol 2015;25:213-25. https://doi.org/10.1002/ima.22139.

[36] Rundo L, Militello C, Russo G, Garufi A, Vitabile S, Gilardi MC, et al. Automated prostate gland segmentation based on an unsupervised fuzzy C-means clustering technique using multispectral T1w and T2w MR imaging. Information 2017;8:49. https://doi.org/10.3390/info8020049.

[37] Rundo L, Stefano A, Militello C, Russo G, Sabini MG, D'Arrigo C, et al. A fully automatic approach for multimodal PET and MR image segmentation in gamma knife treatment planning. Comput Methods Programs Biomed 2017;144:77-96. https://doi.org/10.1016/j.cmpb.2017.03.011.

[38] Militello C, Rundo L, Minafra L, Cammarata FP, Calvaruso M, Conti V, et al. MF2C3: multi-feature fuzzy clustering to enhance cell colony detection in automated clonogenic assay evaluation. Symmetry 2020;12:773. https://doi.org/ $10.3390 /$ sym 12050773.

[39] Comelli A, Stefano A, Russo G, Bignardi S, Sabini MG, Petrucci G, et al. K-nearest neighbor driving active contours to delineate biological tumor volumes. Eng Appl Artif Intell 2019;81:133-44. https://doi.org/10.1016/j.engappai.2019.02.005.

[40] Giannini V, Rosati S, Regge D, Balestra G. Texture Features and Artificial Neural Networks: A Way to Improve the Specificity of a CAD System for Multiparametric MR Prostate Cancer. In: Kyriacou E, Christofides S, Pattichis CS, editors., Cham: Springer International Publishing; 2016, p. 296-301.

[41] Tangaro S, Amoroso N, Brescia M, Cavuoti S, Chincarini A, Errico R, et al. Feature selection based on machine learning in MRIs for hippocampal segmentation. Comput Math Methods Med 2015;2015:814104. https://doi.org/10.1155/2015/ 814104.

[42] Polsinelli M, Cinque L, Placidi G. A light CNN for detecting COVID-19 from CT scans of the chest. Pattern Recognit Lett 2020. https://doi.org/10.1016/j. patrec.2020.10.001.

[43] Bria A, Marrocco C, Tortorella F. Addressing class imbalance in deep learning for small lesion detection on medical images. Comput Biol Med 2020;120:103735. https://doi.org/10.1016/j.compbiomed.2020.103735.

[44] Banzato T, Causin F, Della Puppa A, Cester G, Mazzai L, Zotti A. Accuracy of deep learning to differentiate the histopathological grading of meningiomas on MR images: a preliminary study. J Magn Reson Imaging 2019;50:1152-9. https://doi. org/10.1002/jmri.26723.

45] Bevilacqua V, Brunetti A, Guerriero A, Trotta GF, Telegrafo M, Moschetta M. A performance comparison between shallow and deeper neural networks supervised classification of tomosynthesis breast lesions images. Adv Intell Comput 2019;53:3-19. https://doi.org/10.1016/j.cogsys.2018.04.011.

[46] Bizzego A, Bussola N, Chierici M, Maggio V, Francescatto M, Cima L, et al. Evaluating reproducibility of AI algorithms in digital pathology with DAPPER. PLoS Comput Biol 2019;15:e1006269. https://doi.org/10.1371/journal. pcbi.1006269.

[47] Salvi M, Molinaro L, Metovic J, Patrono D, Romagnoli R, Papotti M, et al. Fully automated quantitative assessment of hepatic steatosis in liver transplants. Comput Biol Med 2020;123:103836. https://doi.org/10.1016/j. compbiomed.2020.103836.

[48] Galbusera F, Niemeyer F, Bassani T, Sconfienza LM, Wilke H-J. Estimating the three-dimensional vertebral orientation from a planar radiograph: Is it feasible? 3rd Int Workshop Spine Load Deform 2020;102:109328. https://doi.org/ 10.1016/j.jbiomech.2019.109328.

[49] Merone M, Sansone C, Soda P. A computer-aided diagnosis system for HEp-2 fluorescence intensity classification. Artif Intell Med 2019;97:71-8. https://doi. org/10.1016/j.artmed.2018.11.002.

[50] Mencattini A, Di Giuseppe D, Comes MC, Casti P, Corsi F, Bertani FR, et al. Discovering the hidden messages within cell trajectories using a deep learning approach for in vitro evaluation of cancer drug treatments. Sci Rep 2020;10:7653. https://doi.org/10.1038/s41598-020-64246-3.

[51] Duggento A, Guerrisi M, Toschi N, Scimeca M, Urbano N, Bonanno E, et al. A random initialization deep neural network for discriminating malignant breast cancer lesions. Annu Int Conf IEEE Eng Med Biol Soc Eng Med Biol Soc Int Conf 2019;2019:912-5. https://doi.org/10.1109/EMBC.2019.8856740.

[52] Brunese L, Mercaldo F AUID- ORCID: 0000-0002-9425-1657, Reginelli A, Santone A. Radiomics for Gleason Score Detection through Deep Learning. LID - E5411 [pii] LID - 10.3390/s20185411 [doi]. Sens Basel Switz JID - 101204366 n.d.

[53] Mushtaq J, Pennella R, Lavalle S, Colarieti A, Steidler S, Martinenghi CMA, et al. Initial chest radiographs and artificial intelligence (AI) predict clinical outcomes 
in COVID-19 patients: analysis of 697 Italian patients. Eur Radiol 2020. https:// doi.org/10.1007/s00330-020-07269-8.

[54] Mendizabal A, Tagliabue E, Brunet J-N, Dall'Alba D, Fiorini P, Cotin S. PhysicsBased Deep Neural Network for Real-Time Lesion Tracking in Ultrasound-Guided Breast Biopsy. In: Miller K, Wittek A, Joldes G, Nash MP, Nielsen PMF, editors. Comput. Biomech. Med., Cham: Springer International Publishing; 2020, p. 33-45.

[55] Rundo L, Han C, Nagano Y, Zhang J, Hataya R, Militello C, et al. USE-Net: Incorporating Squeeze-and-Excitation blocks into U-Net for prostate zonal segmentation of multi-institutional MRI datasets. Neurocomputing 2019;365: 31-43. https://doi.org/10.1016/j.neucom.2019.07.006.

[56] Spampinato C, Palazzo S, Giordano D, Aldinucci M, Leonardi R. Deep learning for automated skeletal bone age assessment in X-ray images. Med Image Anal 2017 36:41-51. https://doi.org/10.1016/j.media.2016.10.010.

[57] Fiorentino MC, Moccia S, Capparuccini M, Giamberini S, Frontoni E. A regression framework to head-circumference delineation from US fetal images. Comput Methods Programs Biomed 2021;198:105771. https://doi.org/10.1016/j. cmpb.2020.105771.

[58] Famouri S, Morra L, Lamberti F. A Deep Learning Approach for Efficient Registration of Dual View Mammography. In: Schilling F-P, Stadelmann T, editors. Artif. Neural Netw. Pattern Recognit., Cham: Springer International Publishing; 2020, p. 162-72. https://doi.org/10.1007/978-3-030-58309-5_13.

[59] Marco B, Leonardo B, Enrico G, Francesco S, Gastone C, Claudia T, et al. Circumventing the Curse of Dimensionality in Magnetic Resonance Fingerprinting through a Deep Learning Approach. ArXiv E-Prints 2018:ar:1811.11477.

[60] Kirienko M, Sollini M, Silvestri G, Mognetti S, Voulaz E, Antunovic L, et al. Convolutional neural networks promising in lung cancer T-parameter assessment on baseline FDG-PET/CT. Contrast Media Mol Imaging 2018;2018:1382309. https://doi.org/10.1155/2018/1382309.

[61] Castiglioni I, Ippolito D, Interlenghi M, Monti CB, Salvatore C, Schiaffino S, et al. Artificial intelligence applied on chest X-ray can aid in the diagnosis of COVID-19 infection: a first experience from Lombardy, Italy. MedRxiv 2020: 2020.04.08.20040907. https://doi.org/10.1101/2020.04.08.20040907.

[62] Brunetti A, Cascarano GD, De Feudis I, Moschetta M, Gesualdo L, Bevilacqua V. Detection and Segmentation of Kidneys from Magnetic Resonance Images in Patients with Autosomal Dominant Polycystic Kidney Disease. In: Huang D-S, Jo K-H, Huang Z-K, editors., Cham: Springer International Publishing; 2019, p. 639-50.

[63] Bevilacqua V, Brunetti A, Cascarano GD, Guerriero A, Pesce F, Moschetta M, et al. A comparison between two semantic deep learning frameworks for the autosomal dominant polycystic kidney disease segmentation based on magnetic resonance images. BMC Med Inform Decis Mak 2019;19:244. https://doi.org/10.1186/ s12911-019-0988-4.

[64] Panic J, Defeudis A, Mazzetti S, Rosati S, Giannetto G, Vassallo L, et al. A convolutional neural network based system for colorectal cancer segmentation on MRI images. 42nd Annu Int Conf IEEE Eng Med Biol Soc EMBC 2020;2020: 1675-8. https://doi.org/10.1109/EMBC44109.2020.9175804.

[65] Giannini V, Defeudis A, Rosati S, Cappello G, Vassallo L, Mazzetti S, et al. Deep learning to segment liver metastases on CT images: impact on a radiomics method to predict response to chemotherapy. IEEE Int Symp Med Meas Appl MeMeA 2020;2020:1-5. https://doi.org/10.1109/MeMeA49120.2020.9137150.

[66] Piantadosi G, Sansone M, Fusco R, Sansone C. Multi-planar 3D breast segmentation in MRI via deep convolutional neural networks. Artif Intell Med 2020;103:101781. https://doi.org/10.1016/j.artmed.2019.101781.

[67] Valvano G, Santini G, Martini N, Ripoli A, Iacconi C, Chiappino D, et al. Convolutional neural networks for the segmentation of microcalcification in mammography imaging. J Healthc Eng 2019;2019:9360941. https://doi.org/ $10.1155 / 2019 / 9360941$.

[68] Nanni L, Lumini A, Ghidoni S, Maguolo G. Stochastic selection of activation layers for convolutional neural networks. Sensors 2020;20:1626. https://doi.org/ $10.3390 / \mathrm{s} 20061626$

[69] Lizzi F, Laruina F, Oliva P, Retico A, Fantacci ME. Residual Convolutional Neura Networks to Automatically Extract Significant Breast Density Features. In: Vento M, Percannella G, Colantonio S, Giorgi D, Matuszewski BJ, Kerdegari H, et al., editors., Cham: Springer International Publishing; 2019, p. 28-35.

[70] Antonio F, Danilo C, Simone S, Michele S, Aurelio U. A multimodal deep network for the reconstruction of T2W MR images. ArXiv E-Prints 2019:ar:1908.03009.

[71] Rocca MA, Anzalone N, Storelli L, Del Poggio A, Cacciaguerra L, Manfredi AA, et al. Deep learning on conventional magnetic resonance imaging improves the diagnosis of multiple sclerosis mimics. Invest Radiol 2020. https://doi.org/ 10.1097/RLI.0000000000000735.

72] Ferrari E, Bosco P, Calderoni S, Oliva P, Palumbo L, Spera G, et al. Dealing with confounders and outliers in classification medical studies: the autism spectrum disorders case study. Artif Intell Med 2020;108:101926. https://doi.org/ 10.1016/j.artmed.2020.101926.

[73] Walsh SLF, Calandriello L, Silva M, Sverzellati N. Deep learning for classifying fibrotic lung disease on high-resolution computed tomography: a case-cohort study. Lancet Respir Med 2018;6:837-45. https://doi.org/10.1016/S2213-2600 (18)30286-8.

[74] Moccia S, Banali R, Martini C, Muscogiuri G, Pontone G, Pepi M, et al. Development and testing of a deep learning-based strategy for scar segmentation on CMR-LGE images. Magn Reson Mater Phys Biol Med 2019;32:187-95. https:// doi.org/10.1007/s10334-018-0718-4.

[75] Brunese L, Mercaldo F, Reginelli A, Santone A. Explainable deep learning for pulmonary disease and coronavirus COVID-19 Detection from X-rays. Comput
Methods Programs Biomed 2020;196:105608. https://doi.org/10.1016/j. cmpb.2020.105608.

[76] Basaia S, Agosta F, Wagner L, Canu E, Magnani G, Santangelo R, et al. Automated classification of Alzheimer's disease and mild cognitive impairment using a single MRI and deep neural networks. NeuroImage Clin 2019;21:101645. https://doi. org/10.1016/j.nicl.2018.101645.

[77] Comes MC, Filippi J, Mencattini A, Casti P, Cerrato G, Sauvat A, et al. Multi-scale generative adversarial network for improved evaluation of cell-cell interactions observed in organ-on-chip experiments. Neural Comput Appl 2020. https://doi. org/10.1007/s00521-020-05226-6.

[78] Barucci A. Adversarial radiomics: the rising of potential risks in medical imaging from adversarial learning. Eur J Nucl Med Mol Imaging 2020. https://doi.org/ 10.1007/s00259-020-04879-8.

[79] Andreini P, Bonechi S, Bianchini M, Mecocci A, Scarselli F. Image generation by GAN and style transfer for agar plate image segmentation. Comput Methods Programs Biomed 2020;184:105268. https://doi.org/10.1016/j. cmpb.2019.105268.

[80] Recenti M, Ricciardi C, Gìslason M, Edmunds K, Carraro U, Gargiulo P. Machine Learning Algorithms Predict Body Mass Index Using Nonlinear Trimodal Regression Analysis from Computed Tomography Scans. In: Henriques J, Neves N, de Carvalho P, editors. XV Mediterr. Conf. Med. Biol. Eng. Comput. MEDICON 2019, Cham: Springer International Publishing; 2020, p. 839-46.

[81] Gitto S, Cuocolo R, Albano D, Chianca V, Messina C, Gambino A, et al. MRI radiomics-based machine-learning classification of bone chondrosarcoma. Eur $\mathrm{J}$ Radiol 2020;128:109043. https://doi.org/10.1016/j.ejrad.2020.109043.

[82] Romeo V, Ricciardi C, Cuocolo R, Stanzione A, Verde F, Sarno L, et al. Machine learning analysis of MRI-derived texture features to predict placenta accreta spectrum in patients with placenta previa. Artif Intell MRI 2019;64:71-6. https:// doi.org/10.1016/j.mri.2019.05.017.

[83] Ricciardi C, Edmunds KJ, Recenti M, Sigurdsson S, Gudnason V, Carraro U, et al. Assessing cardiovascular risks from a mid-thigh CT image: a tree-based machine learning approach using radiodensitometric distributions. Sci Rep 2020;10:2863020-59873-9. https://doi.org/10.1038/s41598-020-59873-9.

[84] Ferraro PM, Agosta F, Riva N, Copetti M, Spinelli EG, Falzone Y, et al. Multimodal structural MRI in the diagnosis of motor neuron diseases. NeuroImage Clin 2017; 16:240-7. https://doi.org/10.1016/j.nicl.2017.08.002.

[85] Gallivanone F, Cava C, Corsi F, Bertoli G, Castiglioni I. In silico approach for the Definition of radiomiRNomic signatures for breast cancer differential diagnosis. Int J Mol Sci 2019;20:5825. https://doi.org/10.3390/ijms20235825.

[86] Vai B, Parenti L, Bollettini I, Cara C, Verga C, Melloni E, et al. Predicting differential diagnosis between bipolar and unipolar depression with multiple kernel learning on multimodal structural neuroimaging. Eur Neuropsychopharmacol 2020;34:28-38. https://doi.org/10.1016/j. euroneuro.2020.03.008.

[87] Retico A, Bosco P, Cerello P, Fiorina E, Chincarini A, Fantacci ME. Predictive models based on support vector machines: whole-brain versus regional analysis of structural MRI in the Alzheimer's disease. J Neuroimaging Off J Am Soc Neuroimaging 2015;25:552-63. https://doi.org/10.1111/jon.12163.

[88] Amoroso N, La Rocca M, Monaco A, Bellotti R, Tangaro S. Complex networks reveal early MRI markers of Parkinson's disease. Med Image Anal 2018;48:12-24. https://doi.org/10.1016/j.media.2018.05.004.

[89] Aslani S, Dayan M, Storelli L, Filippi M, Murino V, Rocca MA, et al. Multi-branch convolutional neural network for multiple sclerosis lesion segmentation. NeuroImage 2019;196:1-15. https://doi.org/10.1016/j. neuroimage. 2019.03.068.

[90] Maggipinto T, Bellotti R, Amoroso N, Diacono D, Donvito G, Lella E, et al. DTI measurements for Alzheimer's classification. Phys Med Biol 2017;62:2361-75. https://doi.org/10.1088/1361-6560/aa5dbe.

[91] Cerveri P, Belfatto A, Baroni G, Manzotti A. Stacked sparse autoencoder networks and statistical shape models for automatic staging of distal femur trochlear dysplasia. Int J Med Robot Comput Assist Surg MRCAS 2018;14:e1947. https:// doi.org/10.1002/rcs.1947.

[92] Tartaglione E, Barbano CA, Berzovini C, Calandri M, Grangetto M. Unveiling COVID-19 from CHEST X-Ray with deep learning: a hurdles race with small data. Int J Environ Res Public Health 2020;17:6933. https://doi.org/10.3390/ ijerph17186933.

[93] Patrini I, Ruperti M, Moccia S, Mattos LS, Frontoni E, De Momi E. Transfer learning for informative-frame selection in laryngoscopic videos through learned features. Med Biol Eng Comput 2020;58:1225-38. https://doi.org/10.1007/ s11517-020-02127-7.

[94] Dimauro G, Ciprandi G, Deperte F, Girardi F, Ladisa E, Latrofa S, et al. Nasal cytology with deep learning techniques. Int J Med Inf 2019;122:13-9. https:// doi.org/10.1016/j.ijmedinf.2018.11.010.

[95] Muscogiuri G, Chiesa M, Trotta M, Gatti M, Palmisano V, Dell'Aversana S, et al. Performance of a deep learning algorithm for the evaluation of CAD-RADS classification with CCTA. Atherosclerosis 2020;294:25-32. https://doi.org/ 10.1016/j.atherosclerosis.2019.12.001.

[96] Zaffino P, Pernelle G, Mastmeyer A, Mehrtash A, Zhang H, Kikinis R, et al. Fully automatic catheter segmentation in MRI with 3D convolutional neural networks: application to MRI-guided gynecologic brachytherapy. Phys Med Biol 2019;64: 165008. https://doi.org/10.1088/1361-6560/ab2f47.

[97] Soomro MH, Coppotelli M, Conforto S, Schmid M, Giunta G, Del Secco L, et al. Automated segmentation of colorectal tumor in 3D MRI using 3D multiscale densely connected convolutional neural network. J Healthc Eng 2019;2019: 1075434. https://doi.org/10.1155/2019/1075434. 
[98] Sena P, Fioresi R, Faglioni F, Losi L, Faglioni G, Roncucci L. Deep learning techniques for detecting preneoplastic and neoplastic lesions in human colorectal histological images. Oncol Lett 2019;18:6101-7. https://doi.org/10.3892/ ol.2019.10928.

[99] Morisi R, Manners DN, Gnecco G, Lanconelli N, Testa C, Evangelisti S, et al. Multiclass parkinsonian disorders classification with quantitative MR markers and graph-based features using support vector machines. Parkinsonism Relat Disord 2018;47:64-70. https://doi.org/10.1016/j.parkreldis.2017.11.343.

[100] Bandini A, Orlandi S, Escalante HJ, Giovannelli F, Cincotta M, Reyes-Garcia CA, et al. Analysis of facial expressions in parkinson's disease through video-based automatic methods. J Neurosci Methods 2017;281:7-20. https://doi.org/ 10.1016/j.jneumeth.2017.02.006.

[101] Peruzzo D, Arrigoni F, Triulzi F, Righini A, Parazzini C, Castellani U. A framework for the automatic detection and characterization of brain malformations: validation on the corpus callosum. Med Image Anal 2016;32:233-42. https://doi. org $/ 10.1016 /$ j.media.2016.05.001.

[102] Nanni L, Lumini A, Zaffonato N. Ensemble based on static classifier selection fo automated diagnosis of mild cognitive impairment. J Neurosci Methods 2018; 302:42-6. https://doi.org/10.1016/j.jneumeth.2017.11.002.

[103] Ugga L, Cuocolo R, Solari D, Guadagno E, D'Amico A, Somma T, et al. Prediction of high proliferative index in pituitary macroadenomas using MRI-based radiomics and machine learning. Neuroradiology 2019;61:1365-73. https://doi. org/10.1007/s00234-019-02266-1.

[104] Bertacchini F, Rizzo R, Bilotta E, Pantano P, Luca A, Mazzuca A, et al. Mid-sagittal plane detection for advanced physiological measurements in brain scans. Physio Meas 2019;40:115009. https://doi.org/10.1088/1361-6579/ab4f2e.

[105] Lombardi A, Guaragnella C, Amoroso N, Monaco A, Fazio L, Taurisano P, et al. Modelling cognitive loads in schizophrenia by means of new functional dynamic indexes. NeuroImage 2019;195:150-64. https://doi.org/10.1016/j. neuroimage.2019.03.055.

[106] Fasano F, Mitolo M, Gardini S, Venneri A, Caffarra P, Pazzaglia F. Combining structural magnetic resonance imaging and visuospatial tests to classify mild cognitive impairment. Curr Alzheimer Res 2018;15:237-46. https://doi.org/ 10.2174/1567205014666171030112339.

[107] Squarcina L, Castellani U, Bellani M, Perlini C, Lasalvia A, Dusi N, et al. Classification of first-episode psychosis in a large cohort of patients using support vector machine and multiple kernel learning techniques. Individ Subj Predict 2017;145:238-45. https://doi.org/10.1016/j.neuroimage.2015.12.007.

[108] Vasta R, Cerasa A, Sarica A, Bartolini E, Martino I, Mari F, et al. The application of artificial intelligence to understand the pathophysiological basis of psychogenic nonepileptic seizures. Epilepsy Behav EB 2018;87:167-72. https://doi.org/ 10.1016/j.yebeh.2018.09.008

[109] Cerasa A, Castiglioni I, Salvatore C, Funaro A, Martino I, Alfano S, et al. Biomarkers of eating disorders using support vector machine analysis of structura neuroimaging data: preliminary results. Behav Neurol 2015;2015. https://doi org/10.1155/2015/924814

[110] Nanni L, Brahnam S, Salvatore C, Castiglioni I. Alzheimer's Disease Neuroimaging Initiative. Texture descriptors and voxels for the early diagnosis of Alzheimer's disease. Artif Intell Med 2019;97:19-26. https://doi.org/10.1016/j. artmed.2019.05.003.

[111] Salvatore C, Cerasa A, Castiglioni I. MRI characterizes the progressive course of $\mathrm{AD}$ and predicts conversion to Alzheimer's Dementia 24 months before probable diagnosis. Front Aging Neurosci 2018;10:135. https://doi.org/10.3389/ fnagi.2018.00135.

[112] Nigro S, Barbagallo G, Bianco MG, Morelli M, Arabia G, Quattrone A, et al. Track density imaging: a reliable method to assess white matter changes in Progressive Supranuclear Palsy with predominant parkinsonism. Parkinsonism Relat Disord 2019;69:23-9. https://doi.org/10.1016/j.parkreldis.2019.10.020.

[113] Kia SM, Pedregosa F, Blumenthal A, Passerini A. Group-level spatio-temporal pattern recovery in MEG decoding using multi-task joint feature learning. J Neurosci Methods 2017;285:97-108. https://doi.org/10.1016/j. jneumeth.2017.05.004.

[114] Lippi M, Gianotti S, Fama A, Casali M, Barbolini E, Ferrari A, et al. Texture analysis and multiple-instance learning for the classification of malignan lymphomas. Comput Methods Programs Biomed 2020;185:105153. https://doi. org/10.1016/j.cmpb.2019.105153.

[115] Tangaro S, Fanizzi A, Amoroso N, Bellotti R. Alzheimer's disease neuroimaging initiative. A fuzzy-based system reveals Alzheimer's disease onset in subjects with Mild cognitive impairment. Phys Medica PM Int J Devoted Appl Phys Med Biol Off J Ital Assoc Biomed Phys AIFB 2017;38:36-44. https://doi.org/10.1016/j. ejmp.2017.04.027.

[116] Pagani M, Nobili F, Morbelli S, Arnaldi D, Giuliani A, Öberg J, et al. Early identification of MCI converting to AD: a FDG PET study. Eur J Nucl Med Mol Imaging 2017;44:2042-52. https://doi.org/10.1007/s00259-017-3761-x.

[117] Salvatore C, Castiglioni I. A wrapped multi-label classifier for the automatic diagnosis and prognosis of Alzheimer's disease. J Neurosci Methods 2018;302: 58-65. https://doi.org/10.1016/j.jneumeth.2017.12.016.

[118] Previtali F, Bertolazzi P, Felici G, Weitschek E. A novel method and software for automatically classifying Alzheimer's disease patients by magnetic resonance imaging analysis. Comput Methods Programs Biomed 2017;143:89-95. https:// doi.org/10.1016/j.cmpb.2017.03.006.

[119] Castellazzi G, Cuzzoni MG, Cotta Ramusino M, Martinelli D, Denaro F, Ricciardi A, et al. A machine learning approach for the differential diagnosis of alzheimer and vascular dementia fed by MRI selected features. Front Neuroinformatics 2020;14:25. https://doi.org/10.3389/fninf.2020.00025.
[120] Salvatore C, Cerasa A, Battista P, Gilardi MC, Quattrone A, Castiglioni I, et al. Magnetic resonance imaging biomarkers for the early diagnosis of Alzheimer's disease: a machine learning approach. Front Neurosci 2015;9:307. https://doi. org/10.3389/fnins.2015.00307.

[121] Romeo V, Maurea S, Cuocolo R, Petretta M, Mainenti PP, Verde F, et al. Characterization of adrenal lesions on unenhanced MRI using texture analysis: a machine-learning approach. J Magn Reson Imaging JMRI 2018;48:198-204. https://doi.org/10.1002/jmri.25954.

[122] Lombardi A, Amoroso N, Diacono D, Monaco A, Logroscino G, De Blasi R, et al. Association between structural connectivity and generalized cognitive spectrum in Alzheimer's disease. Brain Sci 2020;10:879. https://doi.org/10.3390/ brainsci10110879.

[123] De Carli F, Nobili F, Pagani M, Bauckneht M, Massa F, Grazzini M, et al. Accuracy and generalization capability of an automatic method for the detection of typical brain hypometabolism in prodromal Alzheimer disease. Eur J Nucl Med Mol Imaging 2019;46:334-47. https://doi.org/10.1007/s00259-018-4197-7.

[124] Rosati S, Franco P, Fiandra C, Arcadipane F, Silvetti P, Gallio E, et al. Comparison of different classifiers to recognize active bone marrow from CT images. IEEE Int Symp Med Meas Appl MeMeA 2020;2020:1-5. https://doi.org/10.1109/ MeMeA49120.2020.9137173.

[125] Sacca V, Sarica A, Novellino F, Barone S, Tallarico T, Filippelli E, et al. Evaluation of machine learning algorithms performance for the prediction of early multiple sclerosis from resting-state FMRI connectivity data. Brain Imaging Behav 2019; 13:1103-14. https://doi.org/10.1007/s11682-018-9926-9.

[126] Galbusera F, Bassani T, Casaroli G, Gitto S, Zanchetta E, Costa F, et al. Generative models: an upcoming innovation in musculoskeletal radiology? A preliminary test in spine imaging. Eur Radiol Exp 2018;2:29. https://doi.org/10.1186/s41747018-0060-7.

[127] Retico A, Gori I, Giuliano A, Muratori F, Calderoni S. One-class support vector machines identify the language and default mode regions as common patterns of structural alterations in young children with autism spectrum disorders. Front Neurosci 2016;10.

[128] Castaldi E, Aagten-Murphy D, Tosetti M, Burr D, Morrone MC. Effects of adaptation on numerosity decoding in the human brain. NeuroImage $2016 ; 143$ : 364-77. https://doi.org/10.1016/j.neuroimage.2016.09.020.

[129] Marzi C, Giannelli M, Tessa C, Mascalchi M, Diciotti S. Toward a more reliable characterization of fractal properties of the cerebral cortex of healthy subjects during the lifespan. Sci Rep 2020;10:16957. https://doi.org/10.1038/s41598020-73961-w.

[130] Losurdo L, Fanizzi A, Basile TMA, Bellotti R, Bottigli U, Dentamaro R, et al. Radiomics analysis on contrast-enhanced spectral mammography images for breast cancer diagnosis: A pilot study. Entropy 2019:21. https://doi.org/ 10.3390/e21111110.

[131] D'Amico NC, Grossi E, Valbusa G, Rigiroli F, Colombo B, Buscema M, et al. A machine learning approach for differentiating malignant from benign enhancing foci on breast MRI. Eur Radiol Exp 2020;4:5. https://doi.org/10.1186/ s41747-019-0131-4.

[132] Taroni P, Paganoni AM, Ieva F, Pifferi A, Quarto G, Abbate F, et al. Non-invasive optical estimate of tissue composition to differentiate malignant from benign breast lesions: A pilot study. Sci Rep 2017;7.. https://doi.org/10.1038/ srep40683.

[133] Castaldo R, Pane K, Nicolai E, Salvatore M, Franzese M. The impact of normalization approaches to automatically detect radiogenomic phenotypes characterizing breast cancer receptors status. Cancers 2020:12. https://doi.org/ $10.3390 /$ cancers12020518.

[134] De Logu F, Ugolini F, Maio V, Simi S, Cossu A, Massi D, et al. Recognition of cutaneous melanoma on digitized histopathological slides via artificial intelligence algorithm. Front Oncol 2020:10. https://doi.org/10.3389/ fonc. 2020.01559.

[135] Ligabue G, Pollastri F, Fontana F, Leonelli M, Furci L, Giovanella S, et al. Evaluation of the classification accuracy of the kidney biopsy direct immunofluorescence through convolutional neural networks. Clin J Am Soc Nephrol CJASN 2020;15:1445-54. https://doi.org/10.2215/CJN.03210320.

[136] Chiappa V, Bogani G, Interlenghi M, Salvatore C, Bertolina F, Sarpietro G, et al. The Adoption of Radiomics and machine learning improves the diagnostic processes of women with Ovarian MAsses (the AROMA pilot study). J Ultrasound 2020. https://doi.org/10.1007/s40477-020-00503-5.

[137] Granata V, Fusco R, Risi C, Ottaiano A, Avallone A, De Stefano A, et al. Diffusionweighted MRI and diffusion kurtosis imaging to detect RAS mutation in colorectal liver metastasis. Cancers 2020:12. https://doi.org/10.3390/cancers12092420.

[138] Stanzione A, Cuocolo R, Cocozza S, Romeo V, Persico F, Fusco F, et al. Detection of extraprostatic extension of cancer on biparametric MRI combining texture analysis and machine learning: preliminary results. Acad Radiol 2019;26: 1338-44. https://doi.org/10.1016/j.acra.2018.12.025.

[139] Stanzione A, Cuocolo R, Del Grosso R, Nardiello A, Romeo V, Travaglino A, et al. Deep myometrial infiltration of endometrial cancer on MRI: A radiomics-powered machine learning pilot study. Acad Radiol 2020. https://doi.org/10.1016/j. acra.2020.02.028.

[140] Giambelluca D, Cannella R, Vernuccio F, Comelli A, Pavone A, Salvaggio L, et al PI-RADS 3 lesions: role of prostate MRI texture analysis in the identification of prostate cancer. Curr Probl Diagn Radiol 2019. https://doi.org/10.1067/j. cpradiol.2019.10.009.

[141] Gugliandolo SG, Pepa M, Isaksson LJ, Marvaso G, Raimondi S, Botta F, et al. MRIbased radiomics signature for localized prostate cancer: a new clinical tool for cancer aggressiveness prediction? Sub-study of prospective phase II trial on ultra- 
hypofractionated radiotherapy (AIRC IG-13218). Eur Radiol 2020. https://doi. org/10.1007/s00330-020-07105-z.

[142] Rundo L, Militello C, Tangherloni A, Russo G, Vitabile S, Gilardi MC, et al. NeXt for neuro-radiosurgery: a fully automatic approach for necrosis extraction in brain tumor MRI using an unsupervised machine learning technique. Int J Imaging Syst Technol 2018;28:21-37. https://doi.org/10.1002/ima.22253.

[143] Rundo L, Tangherloni A, Cazzaniga P, Nobile MS, Russo G, Gilardi MC, et al. A novel framework for MR image segmentation and quantification by usin MedGA. Comput Methods Programs Biomed 2019;176:159-72. https://doi.org/ 10.1016/j.cmpb.2019.04.016.

[144] Stefano A, Comelli A, Bravatà V, Barone S, Daskalovski I, Savoca G, et al. A preliminary PET radiomics study of brain metastases using a fully automatic segmentation method. BMC Bioinf 2020;21:325.

[145] Lopez Torres E, Fiorina E, Pennazio F, Peroni C, Saletta M, Camarlinghi N, et al Large scale validation of the M5L lung CAD on heterogeneous CT datasets. Med Phys 2015;42:1477-89. https://doi.org/10.1118/1.4907970.

[146] Astorino A, Fuduli A, Veltri P, Vocaturo E. Melanoma detection by means of multiple instance learning. Interdiscip Sci Comput Life Sci 2020;12:24-31. https://doi.org/10.1007/s12539-019-00341-y.

[147] Romeo V, Cuocolo R, Ricciardi C, Ugga L, Cocozza S, Verde F, et al. Prediction of tumor grade and nodal status in oropharyngeal and oral cavity squamous-cell carcinoma using a radiomic approach. Anticancer Res 2020;40:271-80. htt ps://doi.org/10.21873/anticanres.13949.

[148] Stanzione A, Ricciardi C, Cuocolo R, Romeo V, Petrone J, Sarnataro M, et al. MRI radiomics for the prediction of fuhrman grade in clear cell renal cell carcinoma: a machine learning exploratory study. J Digit Imaging 2020;33:879-87. https:// doi.org/10.1007/s10278-020-00336-y.

[149] Martini N, Aimo A, Barison A, Della Latta D, Vergaro G, Aquaro GD, et al. Deep learning to diagnose cardiac amyloidosis from cardiovascular magnetic resonance. J Cardiovasc Magn Reson 2020;22:84. https://doi.org/10.1186/ s12968-020-00690-4.

[150] Piras P, Torromeo C, Re F, Evangelista A, Gabriele S, Esposito G, et al. Left atria trajectory impairment in hypertrophic cardiomyopathy disclosed by geometric morphometrics and parallel transport. Sci Rep 2016;6.. https://doi.org/10.1038/ srep34906.

[151] Bellavia D, Iacovoni A, Agnese V, Falletta C, Coronnello C, Pasta S, et al. Usefulness of regional right ventricular and right atrial strain for prediction of early and late right ventricular failure following a left ventricular assist device implant: a machine learning approach. Int J Artif Organs 2020;43:297-314. https://doi.org/10.1177/0391398819884941.

[152] Cantoni V, Green R, Ricciardi C, Assante R, Zampella E, Nappi C, et al. A machine learning-based approach to directly compare the diagnostic accuracy of myocardial perfusion imaging by conventional and cadmium-zinc telluride SPECT. J Nucl Cardiol Off Publ Am Soc Nucl Cardiol 2020. https://doi.org/ 10.1007/s12350-020-02187-0.

[153] Fantazzini A, Esposito M, Finotello A, Auricchio F, Pane B, Basso C, et al. 3D automatic segmentation of aortic computed tomography angiography combining multi-view 2D convolutional neural networks. Cardiovasc Eng Technol 2020;11: 576-86. https://doi.org/10.1007/s13239-020-00481-z.

[154] Maffei N, Fiorini L, Aluisio G, D’Angelo E, Ferrazza P, Vanoni V, et al. Hierarchical clustering applied to automatic atlas based segmentation of 25 cardiac sub-structures. Phys Medica PM Int J Devoted Appl Phys Med Biol Off J Ital Assoc Biomed Phys AIFB 2020;69:70-80.

[155] Matos J, Paparo F, Mussetto I, Bacigalupo L, Veneziano A, Perugin Bernardi S, et al. Evaluation of novel coronavirus disease (COVID-19) using quantitative lung CT and clinical data: prediction of short-term outcome. Eur Radiol Exp 2020;4:39. https://doi.org/10.1186/s41747-020-00167-0.

[156] Ulivieri FM, Piodi LP, Grossi E, Rinaudo L, Messina C, Tassi AP, et al. The role of carboxy-terminal cross-linking telopeptide of type I collagen, dual x-ray absorptiometry bone strain and Romberg test in a new osteoporotic fracture risk evaluation: A proposal from an observational study. PLoS One 2018;13: e0190477.

[157] Tanzi L, Vezzetti E, Moreno R, Aprato A, Audisio A, Massè A. Hierarchical fracture classification of proximal femur X-Ray images using a multistage Deep Learning approach. Eur J Radiol 2020;133:109373. https://doi.org/10.1016/j. ejrad.2020.109373.

[158] Galbusera F, Niemeyer F, Wilke H-J, Bassani T, Casaroli G, Anania C, et al. Fully automated radiological analysis of spinal disorders and deformities: a deep learning approach. Eur Spine J Off Publ Eur Spine Soc Eur Spinal Deform Soc Eur Sect Cerv Spine Res Soc 2019;28:951-60. https://doi.org/10.1007/s00586-01905944-z.

[159] Scarpa F, Colonna A, Ruggeri A. Multiple-image deep learning analysis for neuropathy detection in corneal nerve images. Cornea 2020;39:342-7. https:// doi.org/10.1097/ICO.0000000000002181.

[160] Lepore D, Ji MH, Pagliara MM, Lenkowicz J, Capocchiano ND, Tagliaferri L, et al. Convolutional neural network based on fluorescein angiography images for retinopathy of prematurity management. Transl Vis Sci Technol 2020;9:37. https://doi.org/10.1167/tvst.9.2.37.
[161] Cordelli E, Maulucci G, De Spirito M, Rizzi A, Pitocco D, Soda P. A decision support system for type 1 diabetes mellitus diagnostics based on dual channel analysis of red blood cell membrane fluidity. Comput Methods Programs Biomed 2018;162:263-71. https://doi.org/10.1016/j.cmpb.2018.05.025.

[162] Casella A, Moccia S, Frontoni E, Paladini D, De Momi E, Mattos LS. Inter-foetus membrane segmentation for TTTS using adversarial networks. Ann Biomed Eng 2020;48:848-59. https://doi.org/10.1007/s10439-019-02424-9.

[163] Talamonti C, Piffer S, Greto D, Mangoni M, Ciccarone A, Dicarolo P, et al. Radiomic and Dosiomic Profiling of Paediatric Medulloblastoma Tumours Treated with Intensity Modulated Radiation Therapy. In: Vento M, Percannella G, Colantonio S, Giorgi D, Matuszewski BJ, Kerdegari H, et al., editors., Cham: Springer International Publishing; 2019, p. 56-64.

[164] Pota M, Scalco E, Sanguineti G, Farneti A, Cattaneo GM, Rizzo G, et al. Early prediction of radiotherapy-induced parotid shrinkage and toxicity based on CT radiomics and fuzzy classification. Artif Intell Med AIME 2015;2017(81):41-53. https://doi.org/10.1016/j.artmed.2017.03.004.

[165] Leccisotti L, Manfrida S, Barone R, Ripani D, Tagliaferri L, Masiello V, et al. The prognostic role of FDG PET/CT before combined radio-chemotherapy in anal cancer patients. Ann Nucl Med 2020;34:65-73. https://doi.org/10.1007/s12149019-01416-y.

[166] Bizzego A, Bussola N, Salvalai D, Chierici M, Maggio V, Jurman G, et al Integrating deep and radiomics features in cancer bioimaging. IEEE Conf Comput Intell Bioinforma Comput Biol CIBCB 2019;2019:1-8. https://doi.org/10.1109/ CIBCB.2019.8791473.

[167] Seregni M, Paganelli C, Lee D, Greer PB, Baroni G, Keall PJ, et al. Motion prediction in MRI-guided radiotherapy based on interleaved orthogonal cine-MRI. Phys Med Biol 2016;61:872-87. https://doi.org/10.1088/0031-9155/61/2/872.

[168] Ermacora D, Pesente S, Pascoli F, Raducci S, Mauro R, Rumeileh IA, et al. Automated computed tomography-ultrasound cross-modality 3-D contouring algorithm for prostate. Ultrasound Med Biol 2015;41:2646-62. https://doi.org/ 10.1016/j.ultrasmedbio.2015.05.025.

[169] Cusumano D, Lenkowicz J, Votta C, Boldrini L, Placidi L, Catucci F, et al. A deep learning approach to generate synthetic CT in low field MR-guided adaptive radiotherapy for abdominal and pelvic cases. Radiother Oncol J Eur Soc Ther Radiol Oncol 2020;153:205-12. https://doi.org/10.1016/j.radonc.2020.10.018.

[170] Guidi G, Maffei N, Meduri B, D’Angelo E, Mistretta GM, Ceroni P, et al. A machine learning tool for re-planning and adaptive RT: A multicenter cohort investigation. Phys Medica Eur J Med Phys 2016;32:1659-66. https://doi.org/10.1016/j. ejmp.2016.10.005.

[171] Guidi G, Maffei N, Vecchi C, Gottardi G, Ciarmatori A, Mistretta GM, et al. Expert system classifier for adaptive radiation therapy in prostate cancer. Australas Phys Eng Sci Med 2017;40:337-48. https://doi.org/10.1007/s13246-017-0535-5.

[172] Guidi G, Maffei N, Vecchi C, Ciarmatori A, Mistretta GM, Gottardi G, et al. A support vector machine tool for adaptive tomotherapy treatments: prediction of head and neck patients criticalities. Phys Medica Eur J Med Phys 2015;31: 442-51. https://doi.org/10.1016/j.ejmp.2015.04.009.

[173] Amoroso N, Errico R, Bruno S, Chincarini A, Garuccio E, Sensi F, et al. Hippocampal unified multi-atlas network (HUMAN): protocol and scale validation of a novel segmentation tool. Phys Med Biol 2015;60:8851-67. https:// doi.org/10.1088/0031-9155/60/22/8851.

[174] Pesapane F, Codari M, Sardanelli F. Artificial intelligence in medical imaging: threat or opportunity? Radiologists again at the forefront of innovation in medicine. Eur Radiol Exp 2018;2:35-018-0061-6. https://doi.org/10.1186/ s41747-018-0061-6.

[175] Collins GS, Reitsma JB, Altman DG, Moons KG. Transparent reporting of a multivariable prediction model for individual prognosis or diagnosis (TRIPOD): the TRIPOD Statement. BMC Med 2015;13:1-014-0241-z. https://doi.org/ 10.1186/s12916-014-0241-z.

[176] Avanzo M, Trianni A, Botta F, Talamonti C, Stasi M, Iori M. Artificial intelligence and the medical physicist: welcome to the machine. Appl Sci 2021;11:1691. https://doi.org/10.3390/app11041691.

[177] The Artificial Intelligence in Medicine (AIM) experiment. Natl Inst Nucl Phys INFN n.d. https://www.ge.infn.it/wordpress/?page_id=1560\&lang=en (accessed November 28, 2020).

[178] D'hulst L, Van Weehaeghe D, Chiò A, Calvo A, Moglia C, Canosa A, et al. Multicenter validation of [(18)F]-FDG PET and support-vector machine discriminant analysis in automatically classifying patients with amyotrophic lateral sclerosis versus controls. Amyotroph Lateral Scler Front Degener 2018;19: 570-7. https://doi.org/10.1080/21678421.2018.1476548.

[179] Tschandl P, Codella N, Akay BN, Argenziano G, Braun RP, Cabo H, et al. Comparison of the accuracy of human readers versus machine-learning algorithms for pigmented skin lesion classification: an open, web-based, international, diagnostic study. Lancet Oncol 2019;20:938-47. https://doi.org/ 10.1016/S1470-2045(19)30333-X.

[180] Filippi M, Tedeschi G, Pantano P, De Stefano N, Zaratin P, Rocca MA, et al. The Italian Neuroimaging Network Initiative (INNI): enabling the use of advanced MRI techniques in patients with MS. Neurol Sci Off J Ital Neurol Soc Ital Soc Clin Neurophysiol 2017;38:1029-38. https://doi.org/10.1007/s10072-017-2903-z. 